\title{
Solvability of Nonstationary Problems for Nonhomogeneous Incompressible Fluids and the Convergence with Vanishing Viscosity
}

\author{
Shigeharu ITOH and Atusi TANI \\ Hirosaki University and Keio University \\ (Communicated by Y. Maeda)
}

\section{Introduction.}

Let $\Omega$ be a bounded or unbounded domain in $\mathbf{R}^{3}$ with a smooth boundary $S$. We consider the system of equations

$$
\left\{\begin{array}{l}
\rho_{t}+v \cdot \nabla \rho=0, \\
\rho\left[v_{t}+(v \cdot \nabla) v\right]+\nabla p=\mu \Delta v+\rho f \\
\operatorname{div} v=0
\end{array}\right.
$$

in $Q_{T}=\Omega \times[0, T], T>0$, where $f(x, t)$ is a given vector field of external forces, while the density $\rho(x, t)$, the velocity vector $v(x, t)$ and the pressure $p(x, t)$ are the unknowns. The viscosity coefficient $\mu$ is assumed to be a nonnegative constant.

This paper consists of two parts. In the first part, Part 1, we solve (1.1) under the following initial-boundary conditions:

If $\mu>0$,

$$
\left\{\begin{array}{l}
\left.v\right|_{S_{T}}=0, \\
\left.\rho\right|_{t=0}=\rho_{0}(x), \\
\left.v\right|_{t=0}=v_{0}(x),
\end{array}\right.
$$

and if $\mu=0$,

$$
\left\{\begin{array}{l}
\left.v \cdot n\right|_{S_{T}}=0 \\
\left.\rho\right|_{t=0}=\rho_{0}(x) \\
\left.v\right|_{t=0}=v_{0}(x)
\end{array}\right.
$$

where $n$ is the unit outward normal to $S$, and $S_{T}=S \times[0, T]$.

In the second part, Part 2, when $\Omega=\mathbf{R}^{3}$, we consider the Cauchy problem (1.1) and 


$$
\left\{\begin{array}{l}
\left.\rho\right|_{t=0}=\rho_{0}(x), \\
\left.v\right|_{t=0}=v_{0}(x),
\end{array}\right.
$$

and establish the uniform convergence of the solution of (1.1) and (1.4) with $\mu>0$ to the one with $\mu=0$ as $\mu \rightarrow 0$.

We will use the classical notations and results of the Sobolev spaces. For $k=0,1,2, \cdots$ and $1 \leq p \leq \infty$,

$$
\begin{gathered}
W_{p}^{k}(\Omega)=\left\{u \in L_{p}(\Omega) ; \sum_{|\alpha| \leq k}\left\|D_{x}^{\alpha} u\right\|_{L_{p}(\Omega)}<\infty\right\}, \\
W_{p}^{2,1}\left(Q_{T}\right)=\left\{u \in L_{p}\left(Q_{T}\right) ;\|u\|_{W_{p}^{2,1}\left(Q_{T}\right)}=\left\|u_{t}\right\|_{L_{p}\left(Q_{T}\right)}+\sum_{|\alpha| \leq 2}\left\|D_{x}^{\alpha} u\right\|_{L_{p}\left(Q_{T}\right)}<\infty\right\},
\end{gathered}
$$

where $D_{x}^{\alpha}=\left(\partial / \partial x_{1}\right)^{\alpha_{1}}\left(\partial / \partial x_{2}\right)^{\alpha_{2}}\left(\partial / \partial x_{3}\right)^{\alpha_{3}}$.

If $u(x, t) \in W_{p}^{2,1}\left(Q_{T}\right)$ and $p>3$, then for any fixed $t \in[0, T]$, the value of $u(x, t)$ belongs to the Slobodetskii-Besov space $W_{p}^{2-2 / p}(\Omega)$ in which the norm is given by

$$
\|u\|_{W_{p}^{2-2 / p(\Omega)}}=\left(\sum_{|\alpha| \leq 1}\left\|D_{x}^{\alpha} u\right\|_{L_{p}(\Omega)}^{p}+\sum_{|\alpha|=1} \int_{\Omega} \int_{\Omega} \frac{\left|D_{x}^{\alpha} u(x)-D_{y}^{\alpha} u(y)\right|^{p}}{|x-y|^{1+p}} d x d y\right)^{1 / p} .
$$

Moreover, we have the inequality

$$
\|u(\cdot, t)\|_{W_{p}^{2-2 / p}(\Omega)} \leq\|u(\cdot, 0)\|_{W_{p}^{2-2 / p}(\Omega)}+\hat{c}\|u\|_{W_{p}^{2,1}\left(Q_{t}\right)},
$$

where the constant $\hat{c}$ does not depend on $t$ (cf. Ladyzhenskaya-Solonnikov-Ural'ceva [7]).

Our theorems related to the unique solvability are the following.

THEOREM 1.1. Let $p>3$ and $\mu>0$. Assume that

$$
\begin{gathered}
\rho_{0}(x) \in C^{0}(\bar{\Omega}), \quad \nabla \rho_{0}(x) \in W_{p}^{1}(\Omega), \quad 0<m \leq \rho_{0}(x) \leq M<\infty, \\
v_{0}(x) \in W_{p}^{2-2 / p}(\Omega),\left.\quad v_{0}\right|_{s}=0, \quad \operatorname{div} v_{0}=0, \\
f(x, t) \in L_{p}\left(Q_{T}\right) .
\end{gathered}
$$

Then there exists $T_{1} \in(0, T]$ such that problem $(1.1),(1.2)$ has a unique solution $(\rho, v, p)(x, t)$ which satisfies

$$
\begin{gathered}
\rho(x, t) \in C^{0}\left(\bar{Q}_{T_{1}}\right), \quad \nabla \rho(x, t) \in C^{0}\left(\left[0, T_{1}\right] ; W_{p}^{1}(\Omega)\right), \\
0<m \leq \rho(x, t) \leq M<\infty, \\
v(x, t) \in W_{p}^{2,1}\left(Q_{T_{1}}\right), \\
\nabla p(x, t) \in L_{p}\left(Q_{T_{1}}\right) .
\end{gathered}
$$

THEOREM 1.2. Let $p>3$ and $\mu=0$. Assume that

$$
\rho_{0}(x) \in C^{0}(\bar{\Omega}), \quad \nabla \rho_{0}(x) \in W_{p}^{1}(\Omega), \quad 0<m \leq \rho_{0}(x) \leq M<\infty,
$$




$$
\begin{gathered}
v_{0}(x) \in W_{p}^{2}(\Omega),\left.\quad v_{0} \cdot n\right|_{s}=0, \quad \operatorname{div} v_{0}=0, \\
f(x, t) \in C^{0}\left([0, T] ; W_{p}^{2}(\Omega)\right) .
\end{gathered}
$$

Then there exists $T_{2} \in(0, T]$ such that problem (1.1), (1.3) has a unique solution $(\rho, v, p)(x, t)$ which satisfies

$$
\begin{gathered}
\rho(x, t) \in C^{0}\left(\bar{Q}_{T_{2}}\right), \quad \nabla \rho(x, t) \in C^{0}\left(\left[0, T_{2}\right] ; W_{p}^{1}(\Omega)\right), \\
0<m \leq \rho(x, t) \leq M<\infty \\
v(x, t) \in C^{0}\left(\left[0, T_{2}\right] ; W_{p}^{2}(\Omega)\right) \\
\nabla p(x, t) \in C^{0}\left(\left[0, T_{2}\right] ; W_{p}^{2}(\Omega)\right) .
\end{gathered}
$$

REMARK. In the case that $\Omega$ is bounded, Theorems 1.1 and 1.2 were proved by Ladyzhenskaya-Solonnikov [6] and Valli-Zajaczkowski [13], respectively. See also $[2-4,8,9]$. However, in the case that $\Omega$ is unbounded, it seems to the authors that the rigorous proofs for these theorems have not been given yet.

The next theorem is concerned with the vanishing viscosity. Analogous result was obtained in [5] in the Sobolev spaces of Hilbert type.

THEOREM 1.3. Let $p \geq 4$ and $0 \leq \mu \leq 1$, and assume that

$$
\begin{gathered}
\rho_{0}(x) \in C^{0}\left(\mathbf{R}^{3}\right), \quad \nabla \rho_{0}(x) \in W_{p}^{1}\left(\mathbf{R}^{3}\right), \quad 0<m \leq \rho_{0}(x) \leq M<\infty, \\
v_{0}(x) \in W_{p}^{2}\left(\mathbf{R}^{3}\right), \quad \operatorname{div} v_{0}=0, \\
f(x, t) \in C^{0}\left([0, T] ; W_{p}^{2}\left(\mathbf{R}^{3}\right)\right) .
\end{gathered}
$$

Then there exists $T_{0} \in(0, T]$ independent of $\mu$ such that problem (1.1), (1.4) has a unique solution $(\rho, v, p)(x, t)$ which satisfies

$$
\begin{gathered}
\rho(x, t) \in C^{0}\left(\mathbf{R}^{3} \times\left[0, T_{0}\right]\right), \quad \nabla \rho(x, t) \in C^{0}\left(\left[0, T_{0}\right] ; W_{p}^{1}\left(\mathbf{R}^{3}\right)\right), \\
0<m \leq \rho(x, t) \leq M<\infty, \\
v(x, t) \in C^{0}\left(\left[0, T_{0}\right] ; W_{p}^{2}\left(\mathbf{R}^{3}\right)\right), \\
\nabla p(x, t) \in C^{0}\left(\left[0, T_{0}\right] ; W_{p}^{1}\left(\mathbf{R}^{3}\right)\right) .
\end{gathered}
$$

Furthermore, let $\left(\rho^{0}, v^{0}, p^{0}\right)$ be the solution of problem (1.1), (1.4) with $\mu=0$ and $\left(\rho^{\mu}, v^{\mu}, p^{\mu}\right)$ the one with $\mu>0$, then we have

$$
\begin{aligned}
\sup _{0 \leq t \leq T_{0}}\left[\left\|\left(\rho^{0}-\rho^{\mu}\right)(t)\right\|_{W_{p}^{1}\left(\mathbf{R}^{3}\right)}\right. & +\left\|\left(v^{0}-v^{\mu}\right)(t)\right\|_{W_{p}^{1}\left(\mathbf{R}^{3}\right)} \\
& \left.+\left\|\nabla\left(p^{0}-p^{\mu}\right)(t)\right\|_{W_{p}^{1}\left(\mathbf{R}^{3}\right)}\right] \rightarrow 0 \quad \text { as } \quad \mu \rightarrow 0 .
\end{aligned}
$$

Part 1 is divided into two sections: In sections 2 and 3, we shall prove Theorems 1.1 and 1.2, respectively. Finally, Theorem 1.3 will be established in Part 2, section 4. 


\section{Part I. Existence Theorems}

\section{The case $\mu>0$.}

In this section, we prove Theorem 1.1 by dividing into three subsections.

2.1. Auxiliary problems. By $C^{\alpha, \beta}\left(\bar{Q}_{T}\right)(0<\alpha<1,0<\beta<1)$, we mean the space of functions which are defined in $\bar{Q}_{T}$ and Hölder continuous with exponent $\alpha$ with respect to $x$ and with exponent $\beta$ with respect to $t$. The norm is

$$
\|u\|_{C^{\alpha, \beta}\left(\bar{Q}_{T)}\right)}=\sup _{\bar{Q}_{T}}|u(x, t)|+[u]_{C^{\alpha, \beta}\left(\bar{Q}_{T}\right)},
$$

where

$$
[u]_{C^{\alpha, \beta}\left(\bar{Q}_{T}\right)}=\sup _{(x, t),(y, s) \in \bar{Q}_{T, x \neq y, t} \neq s} \frac{|u(x, t)-u(y, s)|}{|x-y|^{\alpha}+|t-s|^{\beta}} .
$$

Lemma 2.1. Let $\rho(x, t) \in C^{\alpha, \beta}\left(\bar{Q}_{T}\right), \alpha, \beta \in(0,1)$ such that $0<m \leq \rho(x, t) \leq M<\infty$. Then for any $g(x, t) \in L_{p}\left(Q_{T}\right)$ and $v_{0}(x) \in W_{p}^{2-2 / p}(\Omega)$ with $\left.v_{0}\right|_{s}=0$ and $\operatorname{div} v_{0}=0$, problem

$$
\left\{\begin{array}{l}
\rho v_{t}-\mu \Delta v+\nabla p=g, \\
\operatorname{div} v=0 \\
\left.v\right|_{S_{T}}=0 \\
\left.v\right|_{t=0}=v_{0}(x)
\end{array}\right.
$$

has a unique solution $v(x, t) \in W_{p}^{2,1}\left(Q_{T}\right)$ and $\nabla p(x, t) \in L_{p}\left(Q_{T}\right)$, satisfying

$$
\|v\|_{W_{p}^{2,1}\left(Q_{T}\right)}+\|\nabla p\|_{L_{p}\left(Q_{T}\right)} \leq K_{1}\left(\|\rho\|_{C^{\alpha, \beta}\left(\bar{Q}_{T}\right)}, T\right)\left(\left\|v_{0}\right\|_{W_{p}^{2-2 / p(\Omega)}}+\|g\|_{L_{p}\left(Q_{T}\right)}\right),
$$

where $K_{1}$ is an increasing function of $\|\rho\|_{C^{\alpha, \beta}\left(\bar{Q}_{T}\right)}$ and $T$, depending on $m$ and $M$.

Proof. Let us seek the solution of $(2.1)$ in the form $v(x, t)=u(x, t)+w(x, t)$ and $p(x, t)=r(x, t)+s(x, t)$, where $(u, r)$ and $(w, s)$ satisfy the following systems, respectively:

$$
\left\{\begin{array}{l}
u_{t}-\mu \Delta u+\nabla r=g \\
\operatorname{div} u=0 \\
\left.u\right|_{S_{T}}=0 \\
\left.u\right|_{t=0}=v_{0}(x)
\end{array}\right.
$$

and

$$
\left\{\begin{array}{l}
\rho w_{t}-\mu \Delta w+\nabla s=(1-\rho) u_{t} \equiv g^{\prime} \\
\operatorname{div} w=0 \\
\left.w\right|_{S_{T}}=0 \\
\left.w\right|_{t=0}=0
\end{array}\right.
$$


Problem (2.3) was solved by Solonnikov [11] so that it has a unique solution $u(x, t) \in W_{p}^{2,1}\left(Q_{T}\right)$ and $\nabla r(x, t) \in L_{p}\left(Q_{T}\right)$, satisfying

$$
\|u\|_{W_{p}^{2,1}\left(Q_{T}\right)}+\|\nabla r\|_{L_{p}\left(Q_{T}\right)} \leq c_{1}\left(1+\mathrm{e}^{c_{1} T}\right)\left(\left\|v_{0}\right\|_{W_{p}^{2-2 / p}(\Omega)}+\|g\|_{L_{p}\left(Q_{T}\right)}\right),
$$

where $c_{1}$ is a constant independent of $T$. Therefore it is sufficient to establish the unique solvability of (2.4). It is proved by the method of regularizer. To this end, we introduce two systems of covering $\left\{\omega^{(k)}\right\}$ and $\left\{\Omega^{(k)}\right\}$ such that

1. $\omega^{(k)} \subset \Omega^{(k)} \subset \bar{\Omega}$ and $\bigcup_{k} \omega^{(k)}=\bigcup_{k} \Omega^{(k)}=\bar{\Omega}$,

2. for any $x$, there exists $\omega^{(k)}$ such that $x \in \omega^{(k)}$ and $\operatorname{dist}\left(x, \bar{\Omega}-\omega^{(k)}\right) \geq \delta_{1}>0$,

3. for any $\lambda>0$, there exists a number $N_{0}$ independent of $\lambda$ such that $\bigcap_{k=1}^{N_{0}+1} \Omega^{(k)}=\varnothing$

4. (a) if $\Omega^{(k)} \cap S=\varnothing$ (we denote the set of indices $k$ by $\mathscr{M}$ ), then $\omega^{(k)}$ and $\Omega^{(k)}$ are the cubes with the same center and with the length of their edges equal to $\lambda / 2$ and $\lambda$, respectively,

(b) if $\omega^{(k)} \cap S \neq \varnothing$ (we denote the set of indices $k$ by $\mathscr{N}$ ), then for a local rectangular coordinate system $\{y\}$ with the center $\xi^{(k)} \in S$,

$$
\begin{gathered}
\omega^{(k)}=\left\{\left|y_{i}\right| \leq \frac{1}{2} \delta_{2} \lambda(i=1,2), 0 \leq y_{3}-F\left(y^{\prime} ; \xi^{(k)}\right) \leq \delta_{2} \lambda\right\}, \\
\Omega^{(k)}=\left\{\left|y_{i}\right| \leq \delta_{2} \lambda(i=1,2), 0 \leq y_{3}-F\left(y^{\prime} ; \xi^{(k)}\right) \leq 2 \delta_{2} \lambda\right\},
\end{gathered}
$$

where $F\left(y^{\prime} ; \xi^{(k)}\right)\left(y^{\prime}=\left(y_{1}, y_{2}\right)\right)$ is a function describing the boundary $S$ in the neighborhood of $\xi^{(k)}$ and $\delta_{2}$ is a positive constant independent of $\lambda$.

By changing the variables in such a way that $z_{i}=y_{i}(i=1,2)$ and $z_{3}=y_{3}-F\left(y^{\prime}\right)$, $\Omega^{(k)}(k \in \mathscr{N})$ and the boundary in $\Omega^{(k)}$ are, respectively, transformed into a standard cube

$$
\begin{gathered}
K=\left\{\left|z_{i}\right| \leq \delta_{2} \lambda(i=1,2), 0 \leq z_{3} \leq 2 \delta_{2} \lambda\right\}, \\
K^{\prime}=\left\{\left|z_{i}\right| \leq \delta_{2} \lambda(i=1,2), z_{3}=0\right\} .
\end{gathered}
$$

Furthermore, it is well known that there exist the smooth functions $\left\{\zeta^{(k)}(x)\right\}$ and $\left\{\eta^{(k)}(x)\right\}$ such that

$$
\left\{\begin{array}{l}
\zeta^{(k)}(x)=\left\{\begin{array}{ll}
1 & \text { if } x \in \omega^{(k)}, \\
0 & \text { if } x \in \bar{\Omega}-\Omega^{(k)},
\end{array}, \quad 0 \leq \zeta^{(k)}(x) \leq 1,\right. \\
\eta^{(k)}(x)=0 \quad \text { if } x \in \bar{\Omega}-\Omega^{(k)}, \quad \sum_{k} \zeta^{(k)}(x) \eta^{(k)}(x)=1, \\
\left|D_{x}^{\alpha} \zeta^{(k)}(x)\right| \leq c_{\alpha} \lambda^{-|\alpha|}, \quad\left|D_{x}^{\alpha} \eta^{(k)}(x)\right| \leq c_{\alpha} \lambda^{-|\alpha|} .
\end{array}\right.
$$

Now, let us construct regularizer. For $k \in \mathscr{M}$, let $\left(\bar{w}^{(k)}, \bar{s}^{(k)}\right)(x, t)$ be the solution of problem 


$$
\left\{\begin{array}{l}
\rho\left(\xi^{(k)}, 0\right) \bar{w}_{t}^{(k)}-\mu \Delta \bar{w}^{(k)}+\nabla \bar{s}^{(k)}=\zeta^{(k)}(x) g^{\prime}, \\
\operatorname{div} \bar{w}^{(k)}=0, \\
\left.\bar{w}^{(k)}\right|_{t=0}=0 .
\end{array}\right.
$$

Further, for $k \in \mathscr{N}$, let $\left(\bar{w}^{(k)}, \bar{s}^{(k)}\right)(z, t)$ be the solution of problem

$$
\left\{\begin{array}{l}
\rho\left(\xi^{(k)}, 0\right) \bar{w}_{t}^{(k)}-\mu \Delta \bar{w}^{(k)}+\nabla \bar{s}^{(k)}=\Pi_{z}^{x} \zeta^{(k)}(x) g^{\prime} \\
\operatorname{div} \bar{w}^{(k)}=0 \\
\left.\bar{w}^{(k)}\right|_{z_{n}=0}=0 \\
\left.\bar{w}^{(k)}\right|_{t=0}=0
\end{array}\right.
$$

where $\Pi_{z}^{x}$ is the transformation from $x$ to $z$.

These problems were also solved in [11] to have a unique solution satisfying

$$
\begin{gathered}
\left\|\bar{w}_{t}^{(k)}\right\|_{L_{p}\left(\mathbf{R}_{T}^{3}\right)}+\sum_{|\alpha|=2}\left\|D_{x}^{\alpha} \bar{w}^{(k)}\right\|_{L_{p}\left(\mathbf{R}_{T}^{3}\right)}+\left\|\nabla \bar{s}^{(k)}\right\|_{L_{p}\left(\mathbf{R}_{T}^{3}\right)} \leq c_{2}\left\|\zeta^{(k)} g^{\prime}\right\|_{L_{p}\left(\mathbf{R}_{T}^{3}\right)}, \\
\left\|\nabla \bar{w}^{(k)}\right\|_{L_{p}\left(\mathbf{R}_{T}^{3}\right)}+\left\|\bar{s}^{(k)}\right\|_{L_{p}\left(\mathbf{R}_{T}^{3}\right)} \leq c_{2} \sqrt{T}\left\|\zeta^{(k)} g^{\prime}\right\|_{L_{p}\left(\mathbf{R}_{T}^{3}\right)}, \\
\left\|\bar{w}^{(k)}\right\|_{L_{p}\left(\mathbf{R}_{T}^{3}\right)} \leq c_{2} T\left\|\zeta^{(k)} g^{\prime}\right\|_{L_{p}\left(\mathbf{R}_{T}^{3}\right)}
\end{gathered}
$$

where $\mathbf{R}_{T}^{3}=\left\{\begin{array}{ll}\mathbf{R}^{3} \times[0, T] & \text { for } k \in \mathscr{M} \\ \mathbf{R}_{+}^{3} \times[0, T] & \text { for } k \in \mathscr{N}\end{array}\right.$ and $\mathbf{R}_{+}^{3}=\left\{x \in \mathbf{R}^{3} ; x_{3}>0\right\}$.

Defining the regularizer $R$ by the formula

$$
R h=\sum_{k} \eta^{(k)}(x)\left(w^{(k)}, s^{(k)}\right)(x, t)=\sum_{k} \eta^{(k)}(x) \Pi_{x}^{z}\left(\bar{w}^{(k)}, \bar{s}^{(k)}\right)(z, t)
$$

for $h=\left(g^{\prime}, 0,0\right)$, and the operator $A$ by

$$
A(w, s)=\left(\rho w_{t}-\mu \Delta w+\nabla s, \operatorname{div} w,\left.w\right|_{S_{T}}\right),
$$

we obtain $A R h=h+M h$. Here $M h=\left(M_{1} h, M_{2} h, 0\right)$,

$$
\begin{aligned}
M_{1} h= & \sum_{k}\left[\left\{\rho(x, t)\left(\eta^{(k)} w^{(k)}\right)_{t}-\mu \Delta\left(\eta^{(k)} w^{(k)}\right)+\nabla\left(\eta^{(k)} s^{(k)}\right)\right\}\right. \\
& \left.-\eta^{(k)}\left\{\rho(x, t) w_{t}^{(k)}-\mu \Delta w^{(k)}+\nabla s^{(k)}\right\}\right] \\
& +\sum_{k} \eta^{(k)}\left[\left\{\rho(x, t) w_{t}^{(k)}-\mu \Delta w^{(k)}+\nabla s^{(k)}\right\}-\left\{\rho\left(\xi^{(k)}, 0\right) w_{t}^{(k)}-\mu \Delta w^{(k)}+\nabla s^{(k)}\right\}\right] \\
& +\sum_{k \in \mathcal{N}} \eta^{(k)} \Pi_{x}^{z}\left[\left\{\rho\left(\xi^{(k)}, 0\right) \bar{w}_{t}^{(k)}-\mu\left(\nabla-\nabla F \cdot \nabla_{3}\right)^{2} \bar{w}^{(k)}+\left(\nabla-\nabla F \cdot \nabla_{3}\right) \bar{s}^{(k)}\right\}\right. \\
& \left.\quad-\left\{\rho\left(\xi^{(k)}, 0\right) \bar{w}_{t}^{(k)}-\mu \Delta \bar{w}^{(k)}+\nabla \bar{s}^{(k)}\right\}\right] \\
= & \sum_{k}\left[-2 \mu \nabla \eta^{(k)} \nabla w^{(k)}-\mu \Delta \eta^{(k)} w^{(k)}+\nabla \eta^{(k)} s^{(k)}\right]
\end{aligned}
$$




$$
\begin{aligned}
& +\sum_{k} \eta^{(k)}\left[\rho(x, t)-\rho\left(\xi^{(k)}, 0\right)\right] w_{t}^{(k)} \\
& +\sum_{k \in \mathcal{N}} \eta^{(k)} \Pi_{x}^{z}\left[-\mu\left\{(\nabla F)^{2} \nabla_{3}^{2} \bar{w}^{(k)}-2 \nabla F \nabla_{3} \nabla \bar{w}^{(k)}-\nabla^{2} F \nabla_{3} \bar{w}^{(k)}\right\}-\nabla F \nabla_{3} \nabla_{3} \bar{s}^{(k)}\right], \\
M_{2} h & =\sum_{k}\left[\nabla \cdot\left(\eta^{(k)} w^{(k)}\right)-\eta^{(k)}\left(\nabla \cdot w^{(k)}\right)\right]+\sum_{k \in \mathcal{N}} \eta^{(k)} \Pi_{x}^{z}\left[\left(\nabla-\nabla F \nabla_{3}\right) \cdot \bar{w}^{(k)}-\nabla \cdot \bar{w}^{(k)}\right] \\
& =\sum_{k} \nabla \eta^{(k)} \cdot w^{(k)}-\sum_{k \in \mathcal{N}} \eta^{(k)} \Pi_{x}^{z} \nabla F \nabla_{3} \cdot \bar{w}^{(k)} .
\end{aligned}
$$

It is easily seen that $M$ is a bounded operator in the space

$$
\mathscr{B}_{p, T} \equiv L_{p}\left(Q_{T}\right) \times L_{p}\left(0, T ; W_{p}^{1}(\Omega)\right) \times W_{p}^{2-1 / p}\left(S_{T}\right)
$$

with norm

$$
\|h\|_{\mathscr{B}_{p, T}} \equiv\left\|h_{1}\right\|_{L_{p}\left(Q_{T}\right)}+\left\|h_{2}\right\|_{L_{p}\left(0, T ; W_{p}^{1}(\Omega)\right)}+\left\|h_{3}\right\|_{W_{p}^{2-1 / p}\left(S_{T}\right)}
$$

for $h=\left(h_{1}, h_{2}, h_{3}\right) \in \mathscr{B}_{p, T}$.

If $\tau=\kappa \lambda^{2}$ with $\kappa \leq 1$ and $\kappa \lambda^{2} \leq T$, then by (2.8), (2.9) and (2.10), we get

$$
\begin{aligned}
\left\|M_{1} h\right\|_{L_{p}\left(Q_{\tau}\right)} \leq & c_{3}\left(\frac{\sqrt{\tau}}{\lambda}+\frac{\tau}{\lambda^{2}}+\lambda^{\alpha}+\lambda^{\beta}+\lambda^{2}+\lambda+\sqrt{\tau}\right)\left\|g^{\prime}\right\|_{L_{p}\left(Q_{\tau}\right)} \\
\leq & c_{4}\left(\sqrt{\kappa}+\lambda^{\alpha}+\lambda^{\beta}\right)\left\|g^{\prime}\right\|_{L_{p}\left(Q_{\tau}\right)}, \\
\left\|M_{2} h\right\|_{L_{p}\left(0, \tau ; W_{p}^{1}(\Omega)\right)} & \leq c_{5}\left(\frac{\sqrt{\tau}}{\lambda}+\frac{\tau}{\lambda}+\frac{\tau}{\lambda^{2}}+\lambda \sqrt{\tau}+\lambda+\sqrt{\tau}\right)\left\|g^{\prime}\right\|_{L_{p}\left(Q_{\tau}\right)} \\
& \leq c_{6}(\sqrt{\kappa}+\lambda)\left\|g^{\prime}\right\|_{L_{p}\left(Q_{\tau}\right)},
\end{aligned}
$$

where $c_{4}$ and $c_{6}$ are independent of $\kappa$ and $\lambda$. Therefore we have for sufficiently small $\kappa$ and $\lambda$,

$$
\|M h\|_{\mathscr{B}_{p, \tau}} \leq \frac{1}{2}\|h\|_{\mathscr{B}_{p, \tau}}
$$

Whence the solution $(w, s)$ on the interval $[0, \tau]$ can be found in the form

$$
(w, s)=R\left(I+M+M^{2}+\cdots\right) h=R(I-M)^{-1} h .
$$

Moreover, (2.8), (2.9) and (2.10) imply

$$
\|w\|_{W_{p}^{2,1}\left(Q_{\tau}\right)}+\|\nabla s\|_{L_{p}\left(Q_{\tau}\right)} \leq c_{7}\left\|g^{\prime}\right\|_{L_{p}\left(Q_{\tau}\right)} .
$$

Next we prove the solvability on the interval $[\tau, 2 \tau]$. Put

$$
w^{*}(x, t)= \begin{cases}w(x, t) & \text { for } 0 \leq t \leq \tau, \\ w(x, 2 \tau-t) & \text { for } \tau \leq t \leq 2 \tau,\end{cases}
$$




$$
s^{*}(x, t)= \begin{cases}s(x, t) & \text { for } 0 \leq t \leq \tau, \\ s(x, 2 \tau-t) & \text { for } \tau \leq t \leq 2 \tau .\end{cases}
$$

Let $(\tilde{w}, \tilde{s})(x, t)$ be a solution of problem

$$
\left\{\begin{array}{l}
\rho \tilde{w}_{t}-\mu \Delta \tilde{w}+\nabla \tilde{s}=g^{\prime}(x, t)-g^{\prime}(x, 2 \tau-t) \\
\quad+\rho(x, t) w_{t}(x, 2 \tau-t)+\rho(x, 2 \tau-t) w_{t}(x, 2 \tau-t), \\
\operatorname{div} \tilde{w}=0, \\
\left.\tilde{w}\right|_{\tilde{S}_{\tau}=0,} \\
\left.\tilde{w}\right|_{t=\tau}=0
\end{array}\right.
$$

for $\tau \leq t \leq 2 \tau$, where $\tilde{S}_{\tau}=S \times[\tau, 2 \tau]$, and $(\tilde{w}, \tilde{s})=(0,0)$ for $0 \leq t \leq \tau$. Then it is easy to verify that $w=w^{*}+\tilde{w}$ and $s=s^{*}+\tilde{s}$ is the solution of the problem

$$
\left\{\begin{array}{l}
\rho w_{t}-\mu \Delta w+\nabla s=g^{\prime} \\
\operatorname{div} w=0 \\
\left.w\right|_{s_{2 \tau}}=0 \\
\left.w\right|_{t=0}=0
\end{array}\right.
$$

Repeating this argument, we can obtain the solution on $[0, T]$ satisfying

$$
\|w\|_{W_{p}^{2,1}\left(Q_{T}\right)}+\|\nabla s\|_{L_{p}\left(Q_{T}\right)} \leq c_{8}\left\|g^{\prime}\right\|_{L_{p}\left(Q_{T}\right)} .
$$

From (2.5) and (2.12), we can derive the estimate (2.2).

LEMMA 2.2. If $v(x, t)$ satisfies $\operatorname{div} v=0,\left.v\right|_{S_{T}}=0$ and

$$
\|v\|_{L_{\infty}\left(Q_{T}\right)}+\int_{0}^{T}\|\nabla v(t)\|_{L_{\infty}(\Omega)} d t<\infty,
$$

then for any $\rho_{0}(x) \in C^{1}(\bar{\Omega})$ such that $0<m \leq \rho_{0}(x) \leq M<\infty$, problem

$$
\left\{\begin{array}{l}
\rho_{t}+v \cdot \nabla \rho=0, \\
\left.\rho\right|_{t=0}=\rho_{0}(x),
\end{array}\right.
$$

has a unique solution $\rho(x, t) \in C^{1,1}\left(\bar{Q}_{T}\right)$, which satisfies

$$
\begin{gathered}
m \leq \rho(x, t) \leq M, \\
\|\nabla \rho\|_{L_{\infty}\left(Q_{T}\right)} \leq \sqrt{3}\left\|\nabla \rho_{0}\right\|_{L_{\infty}(\Omega)} \exp \left(\int_{0}^{T}\|\nabla v(t)\|_{L_{\infty}(\Omega)} d t\right), \\
\left\|\rho_{t}\right\|_{L_{\infty}\left(Q_{T}\right)} \leq \sqrt{3}\|v\|_{L_{\infty}\left(Q_{T}\right)}\left\|\nabla \rho_{0}\right\|_{L_{\infty}(\Omega)} \exp \left(\int_{0}^{T}\|\nabla v(t)\|_{L_{\infty}(\Omega)} d t\right) .
\end{gathered}
$$

Moreover, if $\nabla \rho_{0}(x) \in W_{p}^{1}(\Omega)$ and $v(x, t) \in L_{1}\left(0, T ; W_{p}^{2}(\Omega)\right)$, then

$$
\frac{d}{d t}\|\nabla \rho(t)\|_{W_{p}^{1}(\Omega)} \leq c_{9}\|v(t)\|_{W_{p}^{2}(\Omega)}\|\nabla \rho(t)\|_{W_{p}^{1}(\Omega)} .
$$


Proof. It is well-known that, according to the classical method of characteristics, the solution of problem (2.14) is given by $\rho(x, t)=\rho_{0}\left(\left.y(\tau, x, t)\right|_{\tau=0}\right)$, where $y(\tau, x, t)$ is the solution of the Cauchy problem

$$
\left\{\begin{array}{l}
\frac{d y}{d \tau}=v(y, \tau), \\
\left.y\right|_{\tau=t}=x .
\end{array}\right.
$$

From this the estimate (2.15) results. For the estimates (2.16) and (2.17), we refer to Lemma 1.3 in [6].

Next let us establish (2.18). Apply the operator $D_{x}^{\alpha}$ on each side of $(2.14)_{1}$. Multiplying the result by $D_{x}^{\alpha} \rho\left|D_{x}^{\alpha} \rho\right|^{p-2}$, integrating over $\Omega$ and summing over $|\alpha|=1,2$, we have the equality

$$
\begin{aligned}
\frac{1}{p} \frac{d}{d t}\|\nabla \rho(t)\|_{W_{p}^{1}(\Omega)}^{p}= & -\sum_{|\alpha|=1}^{2} \int_{\Omega}\left(v \cdot \nabla D_{x}^{\alpha} \rho\right) D_{x}^{\alpha} \rho\left|D_{x}^{\alpha} \rho\right|^{p-2} d x \\
& -\sum_{|\alpha|=1}^{2} \sum_{0<\beta \leq \alpha}\left(\begin{array}{c}
\alpha \\
\beta
\end{array}\right) \int_{\Omega}\left(D_{x}^{\beta} v \cdot \nabla D_{x}^{\alpha-\beta} \rho\right) D_{x}^{\alpha} \rho\left|D_{x}^{\alpha} \rho\right|^{p-2} d x .
\end{aligned}
$$

The first term of the right hand side is zero, by integration by parts, since $\operatorname{div} v=0$ and $\left.v\right|_{S_{T}}=0$. The second term can be estimated as follows:

$$
\begin{array}{r}
\left.\sum_{|\alpha|=1}^{2} \sum_{0<\beta \leq \alpha}\left(\begin{array}{c}
\alpha \\
\beta
\end{array}\right)\left|\int_{\Omega}\left(D_{x}^{\beta} v \cdot \nabla D_{x}^{\alpha-\beta} \rho\right) D_{x}^{\alpha} \rho\right| D_{x}^{\alpha} \rho\right|^{p-2} d x \mid \\
\leq c_{10}\|\nabla v(t)\|_{W_{p}^{1}(\Omega)}\|\nabla \rho(t)\|_{W_{p}^{1}(\Omega)}^{p} .
\end{array}
$$

Hence we get the estimate (2.18).

The next lemma is directly proved by means of the method of characteristics.

LEMMA 2.3. Let $v(x, t)$ be the same as in Lemma 2.2. If $\rho(x, t) \in C^{1,1}\left(\bar{Q}_{T}\right)$ satisfies

$$
\left\{\begin{array}{l}
\rho_{t}+v \cdot \nabla \rho=\tilde{g} \in L_{1}\left(0, T ; L_{\infty}(\Omega)\right), \\
\left.\rho\right|_{t=0}=0,
\end{array}\right.
$$

then we have

$$
\|\rho(t)\|_{L_{\infty}(\Omega)} \leq \int_{0}^{t}\|\tilde{g}(s)\|_{L_{\infty}(\Omega)} d s .
$$

2.2. Successive approximations. We construct approximate solutions inductively:

$$
v^{(0)}=0
$$


and for $k=1,2,3, \cdots, \rho^{(k)}$ and $\left(v^{(k)}, p^{(k)}\right)$ are, respectively, the solutions of problems

$$
\left\{\begin{array}{l}
\rho_{t}^{(k)}+v^{(k-1)} \cdot \nabla \rho^{(k)}=0, \\
\left.\rho^{(k)}\right|_{t=0}=\rho_{0}(x),
\end{array}\right.
$$

and

$$
\left\{\begin{array}{l}
\rho^{(k)}\left[v_{t}^{(k)}+\left(v^{(k-1)} \cdot \nabla\right) v^{(k-1)}\right]+\nabla p^{(k)}=\mu \Delta v^{(k)}+\rho^{(k)} f \\
\operatorname{div} v^{(k)}=0 \\
v^{(k)} \mid S_{T}=0 \\
\left.v^{(k)}\right|_{t=0}=v_{0}(x)
\end{array}\right.
$$

In this subsection, we show their boundedness.

LEMMA 2.4. For sufficiently small $T_{1} \in(0, T]$, the sequence $\left\{v^{(k)}, \nabla p^{(k)}\right\}_{k}$ is bounded in $W_{p}^{2,1}\left(Q_{T_{1}}\right) \times L_{p}\left(Q_{T_{1}}\right)$.

Proof. Let

$$
V^{(k)}(T)=\left\|v^{(k)}\right\|_{W_{p}^{2,1}\left(Q_{T}\right)}+\left\|\nabla p^{(k)}\right\|_{L_{p}\left(Q_{T}\right)} .
$$

From the consequences in subsection 2.1, we have

$$
\begin{aligned}
& m \leq \rho^{(k)} \leq M, \\
&\left\|\nabla \rho^{(k)}\right\|_{L_{\infty}\left(Q_{T}\right)} \leq \sqrt{3}\left\|\nabla \rho_{0}\right\|_{L_{\infty}(\Omega)} \exp \left(\int_{0}^{T}\left\|\nabla v^{(k-1)}(t)\right\|_{L_{\infty}(\Omega)} d t\right), \\
&\left\|\rho_{t}^{(k)}\right\|_{L_{\infty}\left(Q_{T}\right)} \leq \sqrt{3}\left\|v^{(k-1)}\right\|_{L_{\infty}\left(Q_{T}\right)}\left\|\nabla \rho_{0}\right\|_{L_{\infty}(\Omega)} \exp \left(\int_{0}^{T}\left\|\nabla v^{(k-1)}(t)\right\|_{L_{\infty}(\Omega)} d t\right), \\
& \text { (2.23) }\left\|v^{(k)}\right\|_{W_{P}^{2,1}\left(Q_{T}\right)}+\left\|\nabla p^{(k)}\right\|_{L_{p}\left(Q_{T}\right)} \leq K_{1}\left(\left\|\rho^{(k)}\right\|_{C^{1,1}\left(\bar{Q}_{T}\right)}, T\right) \\
& \times\left(\left\|v_{0}\right\|_{W_{p}^{2-2 / P(\Omega)}}+\left\|\rho^{(k)} f\right\|_{L_{p}\left(Q_{T}\right)}+\left\|\rho^{(k)}\left(v^{(k-1)} \cdot \nabla\right) v^{(k-1)}\right\|_{L_{p}\left(Q_{T}\right)}\right) .
\end{aligned}
$$

Let us estimate the right hand side of (2.23).

First, we can get the inequality

$$
\left\|v^{(k-1)}\right\|_{L_{\infty}\left(Q_{T}\right)} \leq c_{11}\left(\left\|v_{0}\right\|_{W_{p}^{2-2 / p(\Omega)}}+T^{(1-1 / p)(1-3 / p)} V^{(k-1)}(T)\right) .
$$

Indeed, since imbedding theorems imply the inequalities

$$
\begin{aligned}
\left\|v^{(k-1)}(t)-v_{0}\right\|_{L_{\infty}(\Omega)} & \leq c_{12}\left\|v^{(k-1)}(t)-v_{0}\right\|_{W_{p}^{1}(\Omega)}^{3 / p}\left\|v^{(k-1)}(t)-v_{0}\right\|_{L_{p}(\Omega)}^{1-3 / p} \\
\left\|v_{0}\right\|_{L_{\infty}(\Omega)} & \leq c_{12}\left\|v_{0}\right\|_{W_{p}^{1}(\Omega)} \leq c_{13}\left\|v_{0}\right\|_{W_{p}^{2-2 / p}(\Omega)}, \\
\sup _{0 \leq t \leq T}\left\|v^{(k-1)}\right\|_{W_{p}^{1}(\Omega)} & \leq \sup _{0 \leq t \leq T}\left\|v^{(k-1)}\right\|_{W_{p}^{2-2 / p(\Omega)}} \\
& \leq c_{14}\left\|v^{(k-1)}\right\|_{W_{p}^{2,1}\left(Q_{T}\right)}+\left\|v_{0}\right\|_{W_{p}^{2-2 / p}(\Omega)},
\end{aligned}
$$




$$
\begin{aligned}
\left\|v^{(k-1)}(t)-v_{0}\right\|_{L_{p}(\Omega)}^{p} & =\int_{\Omega}\left|v^{(k-1)}(t)-v_{0}\right|^{p} d x=\int_{\Omega}\left|\int_{0}^{t} v_{t}^{(k-1)}(s) d s\right|^{p} d x \\
& \leq \int_{\Omega}\left|\left(\int_{0}^{t} d s\right)^{1 / q}\left(\int_{0}^{t}\left|v_{t}^{(k-1)}(s)\right|^{p} d s\right)^{1 / p}\right|^{p} d x \\
& \leq t^{p / q}\left\|v^{(k-1)}\right\|_{W_{p}^{2,1}\left(Q_{T}\right)}^{p}
\end{aligned}
$$

for $p^{-1}+q^{-1}=1$, the estimate (2.24) is easily derived.

Secondly, we have

$$
\begin{aligned}
\int_{0}^{T}\left\|\nabla v^{(k-1)}(t)\right\|_{L_{\infty}(\Omega)} d t & \leq c_{15} \int_{0}^{T}\left\|v^{(\mathrm{k}-1)}(t)\right\|_{W_{p}^{2}(\Omega)} d t \\
& \leq c_{15}\left(\int_{0}^{T} d t\right)^{1 / q}\left(\int_{0}^{T}\left\|v^{(k-1)}(t)\right\|_{W_{p}^{2}(\Omega)}^{p} d t\right)^{1 / p} \\
& \leq c_{15} T^{1 / q}\left\|v^{(k-1)}\right\|_{W_{p}^{2,1}\left(Q_{T}\right)} \leq c_{15} T^{1 / q} V^{(k-1)}(T) .
\end{aligned}
$$

Thirdly, it is obvious that

$$
\left\|\rho^{(k)} f\right\|_{L_{p}\left(Q_{T}\right)} \leq M\|f\|_{L_{p}\left(Q_{T}\right)} .
$$

Finally, we have the estimate

$$
\begin{aligned}
\left\|\rho^{(k)}\left(v^{(k-1)} \cdot \nabla\right) v^{(k-1)}\right\|_{L_{p}\left(Q_{T}\right)} & \leq c_{16} M T^{(2-3 / p) / p} V^{(k-1)}(T)^{(p-3) /(2 p-3)} \\
\times\left(\left\|v_{0}\right\|_{W_{p}^{2-2 / p}(\Omega)}+T^{(1-1 / p)(1-3 / p)} V^{(k-1)}(T)\right)^{3(p-1) /(2 p-3)} & \leq c_{16} M\left[\left\|v_{0}\right\|_{W_{p}^{2-2 / p}(\Omega)}^{2}+T^{\delta} V^{(k-1)}(T)^{2}\right]
\end{aligned}
$$

with some positive constants $\delta$ and $c_{16} \geq M^{-1}+1$. Indeed, from the inequality

$$
\left\|\nabla v^{(k-1)}(t)\right\|_{L_{p}(\Omega)} \leq\left\|v^{(k-1)}(t)\right\|_{W_{p}^{1}(\Omega)} \leq\left\|v^{(k-1)}(t)\right\|_{W_{p}^{2}(\Omega)}^{a}\left\|v^{(k-1)}(t)\right\|_{L_{\infty}(\Omega)}^{1-a}
$$

with $a=(p-3) /(2 p-3)$, it follows that

$$
\begin{aligned}
\left\|\rho^{(k)}\left(v^{(k-1)} \cdot \nabla\right) v^{(k-1)}\right\|_{L_{p}\left(Q_{T}\right)}^{p} & \leq M^{p}\left\|v^{(k-1)}\right\|_{L_{\infty}\left(Q_{T}\right)}^{p} \int_{0}^{T}\left\|\nabla v^{(k-1)}(t)\right\|_{L_{p}(\Omega)}^{p} d t \\
& \leq c_{17} M^{p}\left\|v^{(k-1)}\right\|_{L_{\infty}\left(Q_{T}\right)}^{p(2-a)} \int_{0}^{T}\left\|v^{(k-1)}(t)\right\|_{W_{p}^{2}(\Omega)}^{a p} d t \\
& \leq c_{17} M^{p}\left\|v^{(k-1)}\right\|_{L_{\infty}\left(Q_{T}\right)}^{p(2-a)} T^{1-a}\left\|v^{(k-1)}\right\|_{W_{p}^{2,1}\left(Q_{T}\right)}^{a p} .
\end{aligned}
$$

This inequality combined with (2.24) leads to (2.27). Moreover, we obtain

$$
\begin{aligned}
\left\|\rho^{(k)}\right\|_{C^{1,1}\left(\bar{Q}_{T}\right)} \leq M & +\sqrt{3}\left(1+\left\|v^{(k-1)}\right\|_{L_{\infty}\left(Q_{T}\right)}\right) \\
& \times\left\|\nabla \rho_{0}\right\|_{L_{\infty}(\Omega)} \exp \left(\int_{0}^{T}\left\|\nabla v^{(k-1)}(t)\right\|_{L_{\infty}(\Omega)} d t\right)
\end{aligned}
$$




$$
\begin{aligned}
& \leq M+\sqrt{3}\left[1+c_{11}\left(\left\|v_{0}\right\|_{W_{p}^{2-2 / p(\Omega)}}+T^{(1-1 / p)(1-3 / p)} V^{(k-1)}(T)\right)\right] \\
& \quad \times\left\|\nabla \rho_{0}\right\|_{L_{\infty}(\Omega)} \exp \left(c_{15} T^{(1-1 / p)} V^{(k-1)}(T)\right) \\
& \equiv K_{3}\left(V^{(k-1)}(T), T\right) .
\end{aligned}
$$

Consequently, we have

$$
\begin{aligned}
& V^{(k)}(T) \leq K_{1}\left(K_{3}\left(V^{(k-1)}(T), T\right), T\right) \\
& \times\left[\left\|v_{0}\right\|_{W_{p}^{2-2 / p}(\Omega)}+M\|f\|_{L_{p}\left(Q_{T}\right)}+c_{16} M\left(\left\|v_{0}\right\|_{W_{p}^{2-2 / p}(\Omega)}^{2}+T^{\delta} V^{(k-1)}(T)^{2}\right)\right] \\
& \leq c_{18} M K_{1}\left(K_{3}\left(V^{(k-1)}(T), T\right), T\right) \\
& \quad \times\left[\left\|v_{0}\right\|_{W_{p}^{2-2 / p}(\Omega)}+\left\|v_{0}\right\|_{W_{p}^{2-2 / p}(\Omega)}^{2}+\|f\|_{L_{p}\left(Q_{T}\right)}+T^{\delta} V^{(k-1)}(T)^{2}\right] .
\end{aligned}
$$

We choose

$$
\begin{aligned}
A_{1} \geq K_{1}(M & \left.+\sqrt{3} e\left[1+c_{11}\left(1+\left\|v_{0}\right\|_{W_{p}^{2-2 / p}(\Omega)}\right)\right]\left\|\nabla \rho_{0}\right\|_{L_{\infty}(\Omega)}, T\right) \\
& \times c_{18} M\left[\left\|v_{0}\right\|_{W_{p}^{2-2 / p(\Omega)}}+\left\|v_{0}\right\|_{W_{p}^{2-2 / p}(\Omega)}^{2}+\|f\|_{L_{p}\left(Q_{T}\right)}+1\right],
\end{aligned}
$$

and define

$$
T_{1}=\min \left\{A_{1}^{-(1-1 / p)^{-1}(1-3 / p)^{-1}}, A_{1}^{-2 / \delta},\left(c_{15} A_{1}\right)^{-(1-1 / p)^{-1}}\right\} .
$$

Then it is easily seen that $V^{(k)}\left(T_{1}\right) \leq A_{1}$ holds provided that $V^{(k-1)}\left(T_{1}\right) \leq A_{1}$. Since

$$
V^{(1)}\left(T_{1}\right) \leq K_{1}\left(M+\left\|\nabla \rho_{0}\right\|_{L_{\infty}(\Omega)}, T_{1}\right)\left(\left\|v_{0}\right\|_{W_{p}^{2-2 / p}(\Omega)}+M\|f\|_{L_{p}\left(Q_{T_{1}}\right)}\right) \leq A_{1},
$$

the assertion of the lemma comes out.

Furthermore, we can immediately get

LEMMA 2.5. For any $k=1,2,3, \cdots$,

$$
\begin{gathered}
\left\|\nabla \rho^{(k)}\right\|_{L_{\infty}\left(Q_{T_{1}}\right)}+\left\|\rho_{t}^{(k)}\right\|_{L_{\infty}\left(Q_{T_{1}}\right)} \leq K_{3}\left(A_{1}, T_{1}\right) \equiv A_{2}, \\
\sup _{0 \leq t \leq T_{1}}\left\|\nabla \rho^{(k)}(t)\right\|_{W_{p}^{1}(\Omega)} \leq\left\|\nabla \rho_{0}\right\|_{W_{p}^{1}(\Omega)} \exp \left(c_{19} A_{1}\left(T_{1}\right)^{(p-1) / p}\right) \equiv A_{3}
\end{gathered}
$$

2.3. Proof of Theorem 1.1. Setting $\sigma^{(k)}=\rho^{(k)}-\rho^{(k-1)}, w^{(k)}=v^{(k)}-v^{(k-1)}$ and $q^{(k)}=p^{(k)}-p^{(k-1)}$, we have

$$
\left\{\begin{array}{l}
\sigma_{t}^{(k)}+v^{(k-1)} \cdot \nabla \sigma^{(k)}=-w^{(k-1)} \cdot \nabla \rho^{(k-1)}, \\
\left.\sigma^{(k)}\right|_{t=0}=0
\end{array}\right.
$$




$$
\left\{\begin{array}{l}
\rho^{(k)} w_{t}^{(k)}-\mu \Delta w^{(k)}+\nabla q^{(k)}=g^{(k)} \\
\operatorname{div} w^{(k)}=0 \\
\left.w^{(k)}\right|_{S_{T}}=0 \\
\left.w^{(k)}\right|_{t=0}=0
\end{array}\right.
$$

where

$$
\begin{aligned}
g^{(k)}= & -\sigma^{(k)}\left[v_{t}^{(k-1)}+\left(v^{(k-1)} \cdot \nabla\right) v^{(k-1)}+f\right] \\
& -\rho^{(k-1)}\left[\left(w^{(k-1)} \cdot \nabla\right) v^{(k-2)}+\left(v^{(k-1)} \cdot \nabla\right) w^{(k-1)}\right]
\end{aligned}
$$

Let

$$
W^{(k)}(t)=\left\|w^{(k)}\right\|_{W_{p}^{2,1}\left(Q_{t}\right)}+\left\|\nabla q^{(k)}\right\|_{L_{p}\left(Q_{t}\right)} .
$$

Then, from Lemma 2.3, it follows that for $t \in\left(0, T_{1}\right]$,

$$
\begin{aligned}
\left\|\sigma^{(k)}(t)\right\|_{L_{\infty}(\Omega)} & \leq A_{2} \int_{0}^{t}\left\|w^{(k-1)}(s)\right\|_{L_{\infty}(\Omega)} d s \\
& \leq c_{20} A_{2} \int_{0}^{t}\left\|w^{(k-1)}\right\|_{W_{p}^{2,1}\left(Q_{s}\right)} d s \\
& \leq c_{21} \int_{0}^{t} W^{(k-1)}(s) d s .
\end{aligned}
$$

Furthermore, we have

$$
\left\|\sigma^{(k)}(t)\right\|_{W_{p}^{1}(\Omega)} \leq c_{22} A_{3} \int_{0}^{t}\left\|w^{(k-1)}(s)\right\|_{W_{p}^{1}(\Omega)} d s .
$$

Here we used the imbeddings

$$
\begin{aligned}
\|u\|_{L_{\infty}(\Omega)} & \leq c_{23}\|u\|_{W_{p}^{1}(\Omega)} \leq c_{23}\|u\|_{W_{p}^{2-2 / p(\Omega)}} \\
& \leq c_{24}\left(\|u(x, 0)\|_{W_{p}^{2-2 / p(\Omega)}}+\|u\|_{W_{p}^{2,1}\left(Q_{t}\right)}\right) .
\end{aligned}
$$

Next, using Lemma 2.4, we can estimate each term in $g^{(k)}$ :

$$
\begin{aligned}
\| \sigma^{(k)} & {\left[v_{t}^{(k-1)}+\left(v^{(k-1)} \cdot \nabla\right) v^{(k-1)}+f\right] \|_{L_{p}\left(Q_{t}\right)}^{p} } \\
& \leq\left\|\sigma^{(k)}\right\|_{L_{\infty}\left(Q_{t}\right)}^{p}\left(\left\|v_{t}^{(k-1)}\right\|_{L_{p}\left(Q_{t}\right)}^{p}+\left\|v^{(k-1)}\right\|_{L_{\infty}\left(Q_{t}\right)}^{p}\left\|\nabla v^{(k-1)}\right\|_{L_{p}\left(Q_{t}\right)}^{p}+\|f\|_{L_{p}\left(Q_{t}\right)}^{p}\right) \\
& \leq\left\|\sigma^{(k)}\right\|_{L_{\infty}\left(Q_{t}\right)}^{p}\left(A_{1}^{p}+c_{25}\left(1+\left\|v_{0}\right\|_{W_{p}^{2-2 / p}(\Omega)}^{p}\right)_{1}^{p}+\|f\|_{L_{p}\left(Q_{t}\right)}^{p}\right), \\
\| \rho^{(k-1)} & {\left[\left(w^{(k-1)} \cdot \nabla\right) v^{(k-2)}+\left(v^{(k-1)} \cdot \nabla\right) w^{(k-1)}\right] \|_{L_{p}\left(Q_{t}\right)}^{p} } \\
& \leq M^{p} \int_{0}^{t} d s \int_{\Omega}\left(\left|\nabla v^{(k-2)}\right|^{p}\left|w^{(k-1)}\right|^{p}+\left|v^{(k-1)}\right|^{p}\left|\nabla w^{(k-1)}\right|^{p}\right) d x
\end{aligned}
$$




$$
\begin{gathered}
\leq M^{p}\left(\sup _{0 \leq s \leq t}\left\|\nabla v^{(k-2)}\right\|_{L_{p}(\Omega)}^{p} \int_{0}^{t}\left\|w^{(k-1)}\right\|_{L_{\infty}(\Omega)}^{p} d s\right. \\
\left.+\left\|v^{(k-1)}\right\|_{L_{\infty}\left(Q_{t}\right)}^{p} \int_{0}^{t}\left\|\nabla w^{(k-1)}\right\|_{L_{p}(\Omega)}^{p} d s\right) \\
\leq c_{26} M^{p}\left(\left\|v^{(k-2)}\right\|_{W_{p}^{2,1}\left(Q_{t}\right)}^{p} \int_{0}^{t}\left\|w^{(k-1)}\right\|_{W_{p}^{2,1}\left(Q_{s}\right)}^{p} d s\right. \\
+\left(1+\left\|v_{0}\right\|_{\left.W_{p}^{2-2 / p(\Omega)}\right)}^{p} \int_{0}^{t}\left\|w^{(k-1)}\right\|_{W_{p}^{2,1}\left(Q_{s}\right)}^{p} d s\right) .
\end{gathered}
$$

Hence, Lemma 2.1 yields

$$
\begin{aligned}
W^{(k)}(t) & \leq c_{27}\left[\int_{0}^{t} W^{(k-1)}(s) d s+\left(\int_{0}^{t} W^{(k-1)}(s)^{p} d s\right)^{1 / p}\right] \\
& \leq c_{28}\left(\int_{0}^{t} W^{(k-1)}(s)^{p} d s\right)^{1 / p},
\end{aligned}
$$

consequently,

$$
W^{(k)}\left(T_{1}\right) \leq c_{28}^{k-1} \frac{\left(T_{1}\right)^{(k-1) / p}}{\Gamma(k)^{1 / p}} W^{(1)}\left(T_{1}\right)
$$

Therefore we find that

$$
\sum_{k=1}^{\infty} W^{(k)}\left(T_{1}\right)<\infty
$$

which implies that the sequence $\left(\rho^{(k)}, v^{(k)}, p^{(k)}\right)(x, t)$ converges to the desired solution $(\rho, v, p)(x, t)$ as $k \rightarrow \infty$.

The uniqueness is proved by making use of the estimates analogous to (2.33) and (2.34).

\section{The case $\mu=0$.}

In this section, we prove Theorem 1.2.

3.1. Auxiliary problems. We assume that $v(x, t) \in C^{0}\left([0, T] ; W_{p}^{2}(\Omega)\right)$ is a given function $\operatorname{such}$ that $\operatorname{div} v=0$ and $\left.v \cdot n\right|_{S_{T}}=0$.

LeMma 3.1. Let $\rho(x, t) \in C^{1,1}([0, T] \times \bar{\Omega})$ such that $0<m \leq \rho(x, t) \leq M<\infty$, $\nabla \rho(x, t) \in C^{0}\left([0, T] ; W_{p}^{1}(\Omega)\right)$ and $f(x, t) \in C^{0}\left([0, T] ; W_{p}^{2}(\Omega)\right)$. Then problem 


$$
\left\{\begin{array}{l}
\operatorname{div}\left(\rho^{-1} \nabla p\right)=\operatorname{div} f-\sum_{i, j=1}^{3} v_{x_{j}}^{i} v_{x_{i}}^{j} \equiv F, \\
\left.\rho^{-1} \frac{\partial p}{\partial n}\right|_{S}=f \cdot n+\sum_{i, j=1}^{3} v^{i} v^{j} \phi^{i j} \equiv G, \quad \phi^{i j}=n_{x_{i}}^{j}
\end{array}\right.
$$

has a unique solution $\nabla p(x, t) \in C^{0}\left([0, T] ; W_{p}^{2}(\Omega)\right)$, satisfying

$$
\|\nabla p(t)\|_{W_{p}^{2}(\Omega)} \leq K_{4}\left(\|\nabla \rho(t)\|_{W_{p}^{1}(\Omega)}\right)\left(\|f(t)\|_{W_{p}^{2}(\Omega)}+\|v(t)\|_{W_{p}^{2}(\Omega)}^{2}\right),
$$

where $K_{4}$ is a nondecreasing function of $\|\nabla \rho(t)\|_{W_{p}^{1}(\Omega)}$, depending on $m$ and $M$. Hereafter, $K_{j}$ 's are functions, having the same properties as $K_{4}$.

ProOF. We first note that $(3.1)_{1}$ comes from applying the divergence operator on both sides of $(1.1)_{2}$, and $(3.1)_{2}$ from taking the scalar product of each side of $(1.1)_{2}$ with $n$ (cf. Temam [12]). It is well-known from the result of Agmon, Douglis and Nirenberg [1] that problem (3.1) is solvable in $W_{p}^{2}(\Omega)$ and the estimate

$$
\|\nabla p(t)\|_{W_{p}^{1}(\Omega)} \leq K_{5}\left(\left\|\rho^{-1}\right\|_{C^{1}(\bar{\Omega})}\right)\left(\|F\|_{L_{p}(\Omega)}+\|G\|_{W_{p}^{1-1 / p}(S)}\right)
$$

is valid. Writing the problem in the form

$$
\left\{\begin{array}{l}
\Delta p=\rho F-\rho \nabla\left(\rho^{-1}\right) \cdot \nabla p \\
\left.\frac{\partial p}{\partial n}\right|_{S}=\rho G
\end{array}\right.
$$

we get

$$
\begin{aligned}
\|\nabla p(t)\|_{W_{p}^{2}(\Omega)} & \leq c_{29}\left(\left\|\rho F-\rho \nabla\left(\rho^{-1}\right) \cdot \nabla p\right\|_{W_{p}^{1}(\Omega)}+\|\rho G\|_{W_{p}^{2-1 / p}(S)}\right) \\
& \leq K_{6}\left(\|\nabla \rho(t)\|_{W_{p}^{1}(\Omega)}\right)\left(\|F\|_{W_{p}^{1}(\Omega)}+\|G\|_{W_{p}^{2-1 / p(S)}}\right) \\
& \leq K_{7}\left(\|\nabla \rho(t)\|_{W_{p}^{1}(\Omega)}\right)\left(\|f(t)\|_{W_{p}^{2}(\Omega)}+\|v(t)\|_{W_{p}^{2}(\Omega)}^{2}\right) .
\end{aligned}
$$

LEMMA 3.2. Let $\rho(x, t)$ and $f(x, t)$ be the same as in Lemma 3.1, and $\nabla p(x, t) \in$ $C^{0}\left([0, T] ; W_{p}^{2}(\Omega)\right)$ be the unique solution of (3.1) guaranteed in Lemma 3.1. Then problem

$$
\left\{\begin{array}{l}
u_{t}+v \cdot \nabla u=-\rho^{-1} \nabla p+f, \\
\left.u\right|_{t=0}=v_{0}(x)
\end{array}\right.
$$

has a unique solution $u(x, t) \in C^{0}\left([0, T] ; W_{p}^{2}(\Omega)\right)$. Moreover, $u(x, t)$ satisfies

$$
\begin{aligned}
\frac{d}{d t}\|u(t)\|_{W_{p}^{2}(\Omega)} \leq & c_{30}\|v(t)\|_{W_{p}^{2}(\Omega)}\|u(t)\|_{W_{p}^{2}(\Omega)} \\
& +K_{8}\left(\|\nabla \rho(t)\|_{W_{p}^{1}(\Omega)}\right)\left(\|f(t)\|_{W_{p}^{2}(\Omega)}+\|v(t)\|_{W_{p}^{2}(\Omega)}^{2}\right) .
\end{aligned}
$$

Proof. Referring to the proof of Lemma 2.2, we should only estimate the term 


$$
\sum_{|\alpha|=0}^{2} \int_{\Omega} D_{x}^{\alpha}\left(f-\rho^{-1} \nabla p\right) \cdot D_{x}^{\alpha} u\left|D_{x}^{\alpha} u\right|^{p-2} d x
$$

Since

$$
\begin{aligned}
\sum_{|\alpha|=0}^{2} & \int_{\Omega}\left|D_{x}^{\alpha}\left(\rho^{-1} \nabla p\right) \| D_{x}^{\alpha} u\right|^{p-1} d x \\
& \leq m^{-1}\|\nabla p\|_{W_{p}^{2}(\Omega)}\|u\|_{W_{p}^{2}(\Omega)}^{p-1}+\left\|\nabla \rho^{-1}\right\|_{W_{p}^{1}(\Omega)}\|\nabla p\|_{W_{p}^{1}(\Omega)}\|\nabla u\|_{W_{p}^{1}(\Omega)}^{p-1} \\
& \leq K_{9}\left(\|\nabla \rho\|_{W_{p}^{1}(\Omega)}\right)\left(\|f\|_{W_{p}^{2}(\Omega)}+\|v\|_{W_{p}^{2}(\Omega)}^{2}\right)\|u\|_{W_{p}^{2}(\Omega)}^{p-1},
\end{aligned}
$$

the desired estimate is obtained.

3.2. Successive approximations. In order to prove Theorem 1.2 , we use the method of successive approximations in the following form:

$$
v^{(0)}=0 \text {, }
$$

and for $k=1,2,3, \cdots, \rho^{(k)}, p^{(k)}$ and $u^{(k)}$ are, respectively, the solutions of problems

$$
\begin{gathered}
\left\{\begin{array}{l}
\rho_{t}^{(k)}+v^{(k-1)} \cdot \nabla \rho^{(k)}=0, \\
\left.\rho^{(k)}\right|_{t=0}=\rho_{0}(x),
\end{array}\right. \\
\left\{\begin{array}{l}
\operatorname{div}\left(\frac{1}{\rho^{(k)}} \nabla p^{(k)}\right)=\operatorname{div} f-\sum_{i, j=1}^{3} v_{x_{j}}^{(k-1), i} v_{x_{i}}^{(k-1), j}, \\
\left.\frac{1}{\rho^{(k)}} \frac{\partial p^{(k)}}{\partial n}\right|_{S}=f \cdot n+\sum_{i, j=1}^{3} v^{(k-1), i} v^{(k-1), j} \phi^{i j},
\end{array}\right.
\end{gathered}
$$

and

$$
\left\{\begin{array}{l}
u_{t}^{(k)}+v^{(k-1)} \cdot \nabla u^{(k)}=-\frac{1}{\rho^{(k)}} \nabla p^{(k)}+f \\
\left.u^{(k)}\right|_{t=0}=v_{0}(x)
\end{array}\right.
$$

Finally, let

$$
v^{(k)}=u^{(k)}-\nabla \psi^{(k)},
$$

where $\psi^{(k)}$ is the solution of problem

$$
\left\{\begin{array}{l}
\Delta \psi^{(k)}=\operatorname{div} u^{(k)}, \\
\left.\frac{\partial \psi^{(k)}}{\partial n}\right|_{S}=u^{(k)} \cdot n .
\end{array}\right.
$$

LEMMA 3.3. The sequence $\left\{v^{(k)}\right\}_{k}$ is bounded in $C^{0}\left(\left[0, T_{2}\right] ; W_{p}^{2}(\Omega)\right)$ for a sufficiently small $T_{2} \in(0, T]$.

Proof. From the consequences in the previous subsections, we can derive 


$$
\begin{gathered}
m \leq \rho^{(k)}(x, t) \leq M \\
\left\|\nabla \rho^{(k)}(t)\right\|_{W_{p}^{1}(\Omega)} \leq\left\|\nabla \rho_{0}\right\|_{W_{p}^{1}(\Omega)} \exp \left(c_{9} \int_{0}^{t}\left\|v^{(k-1)}(s)\right\|_{W_{p}^{2}(\Omega)} d s\right) \\
\left\|\nabla p^{(k)}(t)\right\|_{W_{p}^{2}(\Omega)} \leq K_{4}\left(\left\|\nabla \rho^{(k)}(t)\right\|_{W_{p}^{1}(\Omega)}\right)\left(\|f(t)\|_{W_{p}^{2}(\Omega)}+\left\|v^{(k-1)}(t)\right\|_{W_{p}^{2}(\Omega)}^{2}\right) \\
\left\|u^{(k)}(t)\right\|_{W_{p}^{2}(\Omega)} \leq \exp \left(c_{30} \int_{0}^{t}\left\|v^{(k-1)}(s)\right\|_{W_{p}^{2}(\Omega)} d s\right)\left[\left\|v_{0}\right\|_{W_{p}^{2}(\Omega)}\right. \\
\left.+\int_{0}^{t} K_{8}\left(\left\|\nabla \rho^{(k)}(s)\right\|_{W_{p}^{1}(\Omega)}\right)\left(\|f(s)\|_{W_{p}^{2}(\Omega)}+\left\|v^{(k-1)}(s)\right\|_{W_{p}^{2}(\Omega)}^{2}\right) d s\right]
\end{gathered}
$$

Since

$$
\left\|v^{(k)}(t)\right\|_{W_{p}^{2}(\Omega)} \leq\left\|u^{(k)}(t)\right\|_{W_{p}^{2}(\Omega)}+\left\|\nabla \psi^{(k)}(t)\right\|_{W_{p}^{2}(\Omega)} \leq c_{31}\left\|u^{(k)}(t)\right\|_{W_{p}^{2}(\Omega)},
$$

ultimately, we get

$$
\begin{aligned}
\left\|v^{(k)}(t)\right\|_{W_{p}^{2}(\Omega)} \leq & c_{31} \exp \left(c_{30} \int_{0}^{t}\left\|v^{(k-1)}(s)\right\|_{W_{p}^{2}(\Omega)} d s\right)\left[\left\|v_{0}\right\|_{W_{p}^{2}(\Omega)}\right. \\
& \left.+\int_{0}^{t} K_{8}\left(\left\|\nabla \rho^{(k)}(s)\right\|_{W_{p}^{1}(\Omega)}\right)\left(\|f(s)\|_{W_{p}^{2}(\Omega)}+\left\|v^{(k-1)}(s)\right\|_{W_{p}^{2}(\Omega)}^{2}\right) d s\right] .
\end{aligned}
$$

Let us choose

$$
A_{4} \geq 2 c_{31}\left[\left\|v_{0}\right\|_{W_{p}^{2}(\Omega)}+K_{8}\left(2\left\|\nabla \rho_{0}\right\|_{W_{p}^{1}(\Omega)}\right)\left(T\|f\|_{C^{0}\left([0, T] ; W_{p}^{2}(\Omega)\right)}+1\right)\right],
$$

and define

$$
T_{2}=\min \left\{\left(c_{30} A_{4}\right)^{-1} \log 2, A_{4}^{-2},\left(c_{9} A_{4}\right)^{-1} \log 2\right\} .
$$

Then we find that

$$
\sup _{0 \leq t \leq T_{2}}\left\|v^{(k)}(t)\right\|_{W_{p}^{2}(\Omega)} \leq A_{4}
$$

provided that

$$
\sup _{0 \leq t \leq T_{2}}\left\|v^{(k-1)}(t)\right\|_{W_{p}^{2}(\Omega)} \leq A_{4} .
$$

Therefore by induction we have the assertion of the lemma.

By the direct calculation, we get

LEMMA 3.4. For $k=1,2,3, \cdots$, the estimates 


$$
\begin{aligned}
& \sup _{0 \leq t \leq T_{2}}\left\|\nabla \rho^{(k)}(t)\right\|_{W_{p}^{1}(\Omega)} \leq 2\left\|\nabla \rho_{0}\right\|_{W_{p}^{1}(\Omega)} \equiv A_{5}, \\
& \sup _{0 \leq t \leq T_{2}}\left\|\nabla p^{(k)}(t)\right\|_{W_{p}^{2}(\Omega)} \leq K_{4}\left(A_{5}\right)\left(\|f\|_{C^{0}\left([0, T] ; W_{p}^{2}(\Omega)\right)}+A_{4}^{2}\right) \equiv A_{6}, \\
& \sup _{0 \leq t \leq T_{2}}\left\|\rho_{t}^{(k)}(t)\right\|_{W_{p}^{1}(\Omega)} \leq A_{4} A_{5} \equiv A_{7} \\
& \sup _{0 \leq t \leq T_{2}}\left\|u_{t}^{(k)}(t)\right\|_{W_{p}^{1}(\Omega)} \leq A_{4}^{2}+m^{-1} A_{6}\left(1+m^{-1} A_{5}\right)+\|f\|_{C^{0}\left([0, T] ; W_{p}^{2}(\Omega)\right.} \equiv A_{8}
\end{aligned}
$$

hold.

3.3. Proof of Theorem 1.2. Set $\sigma^{(k)}=\rho^{(k)}-\rho^{(k-1)}, h^{(k)}=u^{(k)}-u^{(k-1)}, q^{(k)}=p^{(k)}-$ $p^{(k-1)}$ and $w^{(k)}=v^{(k)}-v^{(k-1)}$. Then we have

$$
\begin{aligned}
& \left\{\begin{array}{l}
\sigma_{t}^{(k)}+v^{(k-1)} \cdot \nabla \sigma^{(k)}=-w^{(k-1)} \cdot \nabla \rho^{(k-1)}, \\
\left.\sigma^{(k)}\right|_{t=0}=0,
\end{array}\right. \\
& \left\{\begin{aligned}
& \operatorname{div}\left(\frac{1}{\rho^{(k)}} \nabla q^{(k)}\right)= \operatorname{div}\left(\frac{\sigma^{(k)}}{\rho^{(k-1)} \rho^{(k)}} \nabla p^{(k-1)}\right) \\
&-\sum_{i, j=1}^{3}\left(w_{x_{j}}^{(k-1), i} v_{x_{i}}^{(k-1), j}+v_{x_{j}}^{(k-2), i} w_{x_{i}}^{(k-1), j}\right), \\
&\left.\frac{1}{\rho^{(k)}} \frac{\partial q^{(k)}}{\partial n}\right|_{S}=\sum_{i, j=1}^{3}\left(w^{(k-1), i} v^{(k-1), j}+v^{(k-2), i} w^{(k-1), j}\right) \phi^{i j} \\
&-\left.\frac{\sigma^{(k)}}{\rho^{(k-1)} \rho^{(k)}} \frac{\partial p^{(k-1)}}{\partial n}\right|_{S},
\end{aligned}\right.
\end{aligned}
$$

$$
\left\{\begin{array}{l}
h_{t}^{(k)}+\left(v^{(k-1)} \cdot \nabla\right) h^{(k)}+\frac{1}{\rho^{(k)}} \nabla q^{(k)}=-\left(w^{(k-1)} \cdot \nabla\right) u^{(k-1)}+\frac{\sigma^{(k)}}{\rho^{(k-1)} \rho^{(k)}} \nabla p^{(k-1)}, \\
\left.h^{(k)}\right|_{t=0}=0 .
\end{array}\right.
$$

In the same way used for getting the estimates of $\rho, p$ and $u$, we get

$$
\begin{gathered}
\left\|\sigma^{(k)}(t)\right\|_{W_{p}^{1}(\Omega)} \leq c_{32} A_{5} \exp \left(c_{32} A_{4} T_{2}\right) \int_{0}^{t}\left\|w^{(k-1)}(s)\right\|_{W_{p}^{1}(\Omega)} d s \\
\equiv A_{9} \int_{0}^{t}\left\|w^{(k-1)}(s)\right\|_{W_{p}^{1}(\Omega)} d s \\
\left\|\nabla q^{(k)}(t)\right\|_{W_{p}^{1}(\Omega)} \leq A_{10}\left(\left\|\sigma^{(k)}(t)\right\|_{W_{p}^{1}(\Omega)}+\left\|w^{(k-1)}(t)\right\|_{W_{p}^{1}(\Omega)}\right), \\
\left\|h^{(k)}(t)\right\|_{W_{p}^{1}(\Omega)} \leq A_{11} \int_{0}^{t}\left(\left\|\sigma^{(k)}(s)\right\|_{W_{p}^{1}(\Omega)}+\left\|\nabla q^{(k)}(s)\right\|_{W_{p}^{1}(\Omega)}+\left\|w^{(k-1)}(s)\right\|_{W_{p}^{1}(\Omega)}\right) d s .
\end{gathered}
$$

From these inequalities, since 


$$
\left\|w^{(k)}(t)\right\|_{W_{p}^{1}(\Omega)} \leq c_{33}\left\|h^{(k)}(t)\right\|_{W_{p}^{1}(\Omega)},
$$

it follows that

$$
\begin{aligned}
\left\|W^{(k)}(t)\right\|_{W_{p}^{1}(\Omega)} & \leq A_{12} \int_{0}^{t}\left\|w^{(k-1)}(s)\right\|_{W_{p}^{1}(\Omega)} d s \\
& \leq A_{12}^{k-1} \frac{t^{k-1}}{(k-1) !} \sup _{0 \leq s \leq t}\left\|w^{(1)}(s)\right\|_{W_{p}^{1}(\Omega)},
\end{aligned}
$$

consequently,

$$
\sup _{0 \leq t \leq T_{2}}\left\|w^{(k)}(t)\right\|_{W_{p}^{1}(\Omega)} \leq A_{4} A_{12}^{k-1} \frac{\left(T_{2}\right)^{k-1}}{(k-1) !} .
$$

Therefore we find that

$$
\sum_{k=1}^{\infty}\left\|w^{(k)}\right\|_{C^{0}\left(\left[0, T_{2}\right] ; W_{p}^{1}(\Omega)\right)}<\infty
$$

This implies that $\left(\rho^{(k)}, p^{(k)}, u^{(k)}, v^{(k)}\right)(x, t) \rightarrow(\rho, p, u, v)(x, t)$ as $k \rightarrow \infty$, which satisfies equations

$$
\begin{aligned}
& \left\{\begin{array}{l}
\rho_{t}+v \cdot \nabla \rho=0 \\
\operatorname{div}\left((v \cdot \nabla) v+\rho^{-1} \nabla p-f\right)=0, \\
u_{t}+(v \cdot \nabla) u+\rho^{-1} \nabla p=f, \\
\Delta \psi=\operatorname{div} u \\
v=u-\nabla \psi
\end{array}\right. \\
& \left\{\begin{array}{l}
\left.\left((v \cdot \nabla) v+\rho^{-1} \nabla p-f\right) \cdot n\right|_{S_{T_{2}}}=0 \\
\left.(u-\nabla \psi) \cdot n\right|_{S_{T_{2}}}=0 \\
\left.\rho\right|_{t=0}=\rho_{0}(x) \\
\left.u\right|_{t=0}=v_{0}(x)
\end{array}\right.
\end{aligned}
$$

Now let us show that $u=v$. Applying the divergence operator on both sides of $(3.15)_{3}$ and taking into account $(3.15)_{2}$, we get

$$
(\operatorname{div} u)_{t}+v \cdot \nabla(\operatorname{div} u)=-\sum_{i, j=1}^{3} v_{x_{j}}^{i} \psi_{x_{i} x_{j}} .
$$

If we take the scalar product of each side of $(3.15)_{3}$ with $n$, we obtain

$$
(u \cdot n)_{t}+v \cdot \nabla(u \cdot n)=\sum_{i, j=1}^{3} v^{i} \psi_{x_{j}} \phi^{i j} .
$$

Noting that $\operatorname{div} v=0,\left.v \cdot n\right|_{S}=0$ and

$$
\|\psi\|_{W_{p}^{2}(\Omega)} \leq c_{34}\left(\|\operatorname{div} u\|_{L_{p}(\Omega)}+\|u \cdot n\|_{W_{p}^{1-1 / p}(S)}\right),
$$


we have the inequality

$$
\|\operatorname{div} u\|_{L_{p}(\Omega)}+\|u \cdot n\|_{W_{p}^{1-1 / p}(S)} \leq c_{35} \int_{0}^{t}\left(\|\operatorname{div} u\|_{L_{p}(\Omega)}+\|u \cdot n\|_{W_{p}^{1-1 / p}(S)}\right) d s,
$$

which means $\operatorname{div} u=0$ and $\left.u \cdot n\right|_{s}=0$.

This completes the proof of Theorem.

\section{Part II. Vanishing Viscosity}

4. The convergence problem as $\mu \rightarrow 0$.

In this section, we shall prove Theorem 1.3.

4.1. A priori estimates. Let $(\rho, v, p)(x, t)$ be a sufficiently regular solution. Hereafter $C$ stands for the generic constant independent of $\mu$.

LeMma 4.1. For $\rho(x, t)$, the estimates

$$
\begin{aligned}
& m \leq \rho(x, t) \leq M, \\
& \frac{d}{d t}\|\nabla \rho(t)\|_{W_{p}^{1}\left(\mathbf{R}^{3}\right)} \leq C\|v(t)\|_{W_{p}^{2}\left(\mathbf{R}^{3}\right)}\|\nabla \rho(t)\|_{W_{p}^{1}\left(\mathbf{R}^{3}\right)}
\end{aligned}
$$

hold. Moreover, if we put $\xi(x, t)=\rho(x, t)^{-1}$, then the estimates

$$
\begin{aligned}
& M^{-1} \leq \xi(x, t) \leq m^{-1}, \\
& \frac{d}{d t}\|\nabla \xi(t)\|_{W_{p}^{1}\left(\mathbf{R}^{3}\right)} \leq C\|v(t)\|_{W_{p}^{2}\left(\mathbf{R}^{3}\right)}\|\nabla \xi(t)\|_{W_{p}^{1}\left(\mathbf{R}^{3}\right)}
\end{aligned}
$$

are valid.

Proof. Quite similarly to the proof of Lemma 2.2, we can obtain (4.1) and (4.2). If we note that $\xi(x, t)$ satisfies the equation

$$
\left\{\begin{array}{l}
\xi_{t}+v \cdot \nabla \xi=0, \\
\left.\xi\right|_{t=0}=\rho_{0}(x)^{-1} \equiv \xi_{0}(x),
\end{array}\right.
$$

the estimates (4.3) and (4.4) directly follow from (4.1) and (4.2).

LemMA 4.2. Let $\omega(x, t)=\operatorname{rot} v(x, t)$. Then the estimate

$$
\begin{aligned}
\frac{d}{d t}\|\omega(t)\|_{W_{p}^{1}\left(\mathbf{R}^{3}\right)} \leq C & \left(1+\|\operatorname{rot} f\|_{C^{0}\left([0, T] ; W_{p}^{1}\left(\mathbf{R}^{3}\right)\right)}\right) \\
& \times\left(1+\|\omega(t)\|_{W_{p}^{1}\left(\mathbf{R}^{3}\right)}+\|\nabla \xi(t)\|_{W_{p}^{1}\left(\mathbf{R}^{3}\right)}+\|\nabla p(t)\|_{W_{p}^{1}\left(\mathbf{R}^{3}\right)}\right)^{3}
\end{aligned}
$$

is valid. 
ProOF. By applying the rotation operator on both sides of $(1.1)_{2}$, we obtain

$$
\omega_{t}+(v \cdot \nabla) \omega-(\omega \cdot \nabla) v+\nabla \xi \times \nabla p=\mu \xi \Delta \omega+\mu \nabla \xi \times \Delta v+\operatorname{rot} f .
$$

If we apply the operator $D_{x}^{\alpha}$ to (4.6), multiply the result by $D_{x}^{\alpha} \omega\left|D_{x}^{\alpha} \omega\right|^{p-2}$, integrate over $\mathbf{R}^{3}$ and sum over $|\alpha|=0,1$, then we have the equality

$$
\begin{aligned}
& \frac{1}{p} \frac{d}{d p}\|\omega(t)\|_{W_{p}^{1}\left(\mathbf{R}^{3}\right)}^{p}=\sum_{|\alpha|=0,1}\left[-\int_{\mathbf{R}^{3}} D_{x}^{\alpha}((v \cdot \nabla) \omega) \cdot D_{x}^{\alpha} \omega\left|D_{x}^{\alpha} \omega\right|^{p-2} d x\right. \\
& \quad+\int_{\mathbf{R}^{3}} D_{x}^{\alpha}((\omega \cdot \nabla) v) \cdot D_{x}^{\alpha} \omega\left|D_{x}^{\alpha} \omega\right|^{p-2} d x-\int_{\mathbf{R}^{3}} D_{x}^{\alpha}(\nabla \xi \times \nabla p) \cdot D_{x}^{\alpha} \omega\left|D_{x}^{\alpha} \omega\right|^{p-2} d x \\
& \quad+\mu \int_{\mathbf{R}^{3}} D_{x}^{\alpha}(\xi \Delta \omega) \cdot D_{x}^{\alpha} \omega\left|D_{x}^{\alpha} \omega\right|^{p-2} d x+\mu \int_{\mathbf{R}^{3}} D_{x}^{\alpha}(\nabla \xi \times \Delta v) \cdot D_{x}^{\alpha} \omega\left|D_{x}^{\alpha} \omega\right|^{p-2} d x \\
& \left.\quad+\int_{\mathbf{R}^{3}} D_{x}^{\alpha} \operatorname{rot} f \cdot D_{x}^{\alpha} \omega\left|D_{x}^{\alpha} \omega\right|^{p-2} d x\right] \equiv \sum_{j=1}^{6} I_{j} .
\end{aligned}
$$

Let us estimate each $I_{j}$ by making use of $\operatorname{div} v=0$ and $\mu \leq 1$.

$$
\begin{aligned}
\left|I_{1}\right|= & \left.\left|\sum_{|\alpha|=0,1} \int_{\mathbf{R}^{3}}(v \cdot \nabla) D_{x}^{\alpha} \omega \cdot D_{x}^{\alpha} \omega\right| D_{x}^{\alpha} \omega\right|^{p-2} d x \\
& +\sum_{|\alpha|=1} \int_{\mathbf{R}^{3}}\left(D_{x}^{\alpha} v \cdot \nabla\right) \omega \cdot D_{x}^{\alpha} \omega\left|D_{x}^{\alpha} \omega\right|^{p-2} d x \mid \\
= & \left.\left|\sum_{|\alpha|=1} \int_{\mathbf{R}^{3}}\left(D_{x}^{\alpha} v \cdot \nabla\right) \omega \cdot D_{x}^{\alpha} \omega\right| D_{x}^{\alpha} \omega\right|^{p-2} d x\left|\leq \sum_{|\alpha|=1} \int_{\mathbf{R}^{3}}\right| D_{x}^{\alpha} v \|\left. D_{x}^{\alpha} \omega\right|^{p} d x \\
\leq & C\left(\sum_{|\alpha|=1}\left\|D_{x}^{\alpha} v(t)\right\|_{W_{p}^{1}\left(\mathbf{R}^{3}\right)}\right)\|\omega(t)\|_{W_{p}^{1}\left(\mathbf{R}^{3}\right)}^{p} \cdot \\
\left|I_{2}\right|= & \left.\left|\sum_{|\alpha|=0,1} \int_{\mathbf{R}^{3}}\left(D_{x}^{\alpha} \omega \cdot \nabla\right) v \cdot D_{x}^{\alpha} \omega\right| D_{x}^{\alpha} \omega\right|^{p-2} d x \\
& +\sum_{|\alpha|=1} \int_{\mathbf{R}^{3}}(\omega \cdot \nabla) D_{x}^{\alpha} v \cdot D_{x}^{\alpha} \omega\left|D_{x}^{\alpha} \omega\right|^{p-2} d x \mid \\
\leq & \sum_{|\alpha|=0,1|\beta|=1} \sum_{\mathbf{R}^{3}}\left|D_{x}^{\beta} v\right|\left|D_{x}^{\alpha} \omega\right|^{p} d x \\
& +\sum_{|\alpha|=1} \sum_{|\beta|=2} \int_{\mathbf{R}^{3}}\left|D_{x}^{\beta} v\|\omega\| D_{x}^{\alpha} \omega\right|^{p-1} d x \\
\leq & C\left(\sum_{|\alpha|=1}\left\|D_{x}^{\alpha} v(t)\right\|_{W_{p}^{1}\left(\mathbf{R}^{3}\right)}\right)\|\omega(t)\|_{W_{p}^{1}\left(\mathbf{R}^{3}\right)}^{p} \cdot
\end{aligned}
$$




$$
\begin{aligned}
& \left|I_{3}\right|=\left.\left|\sum_{|\alpha|=0,1} \int_{\mathbf{R}^{3}}\left(\nabla \xi \times \nabla D_{x}^{\alpha} p\right) \cdot D_{x}^{\alpha} \omega\right| D_{x}^{\alpha} \omega\right|^{p-2} d x \\
& +\sum_{|\alpha|=1} \int_{\mathbf{R}^{3}}\left(\nabla D_{x}^{\alpha} \xi \times \nabla p\right) \cdot D_{x}^{\alpha} \omega\left|D_{x}^{\alpha} \omega\right|^{p-2} d x \mid \\
& \leq 2 \sum_{|\alpha|=0,1} \int_{\mathbf{R}^{3}}\left|\nabla \xi\left\|\nabla D_{x}^{\alpha} p\right\| D_{x}^{\alpha} \omega\right|^{p-1} d x \\
& +2 \sum_{|\alpha|=1} \int_{\mathbf{R}^{3}}\left|\nabla D_{x}^{\alpha} \xi\right|\left|\nabla p \| D_{x}^{\alpha} \omega\right|^{p-1} d x \\
& \leq C\|\nabla \xi(t)\|_{W_{p}^{1}\left(\mathbf{R}^{3}\right)}\|\nabla p(t)\|_{W_{p}^{1}\left(\mathbf{R}^{3}\right)}\|\omega(t)\|_{W_{p}^{1}\left(\mathbf{R}^{3}\right)}^{p-1} . \\
& \begin{aligned}
I_{4}= & \mu \sum_{|\alpha|=0,1} \int_{\mathbf{R}^{3}} \xi \Delta D_{x}^{\alpha} \omega \cdot D_{x}^{\alpha} \omega\left|D_{x}^{\alpha} \omega\right|^{p-2} d x \\
& +\mu \sum_{|\alpha|=1} \int_{\mathbf{R}^{3}} D_{x}^{\alpha} \xi \Delta \omega \cdot D_{x}^{\alpha} \omega\left|D_{x}^{\alpha} \omega\right|^{p-2} d x \equiv I_{4,1}+I_{4,2},
\end{aligned} \\
& I_{4,1}=-\mu \sum_{|\alpha|=0,1} \sum_{|\beta|=1} \int_{\mathbf{R}^{3}} \xi\left|D_{x}^{\alpha} D_{x}^{\beta} \omega\right|^{2}\left|D_{x}^{\alpha} \omega\right|^{p-2} d x \\
& -\mu(p-2) \sum_{|\alpha|=0,1} \sum_{|\beta|=1} \int_{\mathbf{R}^{3}} \xi\left|D_{x}^{\alpha} \omega \cdot D_{x}^{\alpha} D_{x}^{\beta} \omega\right|^{2}\left|D_{x}^{\alpha} \omega\right|^{p-4} d x \\
& -\mu \sum_{|\alpha|=0,1} \sum_{|\beta|=1} \int_{\mathbf{R}^{3}} D_{x}^{\beta} \xi D_{x}^{\alpha} D_{x}^{\beta} \omega \cdot D_{x}^{\alpha} \omega\left|D_{x}^{\alpha} \omega\right|^{p-2} d x .
\end{aligned}
$$

Let the third term of the right hand side be $I_{4,3}$, then

$$
\begin{aligned}
\left|I_{4,2}+I_{4,3}\right| \leq & C \mu \sum_{|\alpha|=0,1} \sum_{|\beta|=1} \int_{\mathbf{R}^{3}}\left|D_{x}^{\beta} \xi\right|\left|D_{x}^{\alpha} D_{x}^{\beta} \omega\right|\left|D_{x}^{\alpha} \omega\right|^{p-1} d x \\
\leq & \frac{\mu}{4 M} \sum_{|\alpha|=0,1} \sum_{|\beta|=1} \int_{\mathbf{R}^{3}}\left|D_{x}^{\alpha} D_{x}^{\beta} \omega\right|^{2}\left|D_{x}^{\alpha} \omega\right|^{p-2} d x \\
& +C \sum_{|\alpha|=0,1} \sum_{|\beta|=1} \int_{\mathbf{R}^{3}}\left|D_{x}^{\beta} \xi\right|^{2}\left|D_{x}^{\alpha} \omega\right|^{p} d x \\
\leq & \frac{\mu}{4 M} \sum_{|\alpha|=0,1} \sum_{|\beta|=1} \int_{\mathbf{R}^{3}}\left|D_{x}^{\alpha} D_{x}^{\beta} \omega\right|^{2}\left|D_{x}^{\alpha} \omega\right|^{p-2} d x \\
& +C\|\nabla \xi(t)\|_{W_{p}^{1}\left(\mathbf{R}^{3}\right)}\|\omega(t)\|_{W_{p}^{1}\left(\mathbf{R}^{3}\right)}^{p} \\
I_{5}=\mu \int_{\mathbf{R}^{3}}(\nabla \xi \times \Delta v) \cdot \omega|\omega|^{p-2} d x & \\
+ & \mu \sum_{|\alpha|=1} \int_{\mathbf{R}^{3}} D_{x}^{\alpha}(\nabla \xi \times \Delta v) \cdot D_{x}^{\alpha} \omega\left|D_{x}^{\alpha} \omega\right|^{p-2} d x \equiv I_{5,1}+I_{5,2},
\end{aligned}
$$




$$
\begin{aligned}
\left|I_{5,1}\right| & \leq 2 \mu \int_{\mathbf{R}^{3}}|\nabla \xi\|\Delta v\| \omega|^{p-1} d x \\
& \leq C\|\nabla \xi(t)\|_{W_{p}^{1}\left(\mathbf{R}^{3}\right)}\|\Delta v(t)\|_{L_{p}\left(\mathbf{R}^{3}\right)}\|\omega(t)\|_{L_{p}\left(\mathbf{R}^{3}\right)}^{p-1}
\end{aligned}
$$

Since

$$
\begin{aligned}
I_{5,2}= & -\mu \sum_{|\alpha|=1} \int_{\mathbf{R}^{3}}(\nabla \xi \times \Delta v) \cdot D_{x}^{2 \alpha} \omega\left|D_{x}^{\alpha} \omega\right|^{p-2} d x \\
& -\mu(p-2) \sum_{|\alpha|=1} \int_{\mathbf{R}^{3}}\left[(\nabla \xi \times \Delta v) \cdot D_{x}^{\alpha} \omega\right] D_{x}^{2 \alpha} \omega \cdot D_{x}^{\alpha} \omega\left|D_{x}^{\alpha} \omega\right|^{p-4} d x
\end{aligned}
$$

we get

$$
\begin{aligned}
\left|I_{5,2}\right| \leq & C \mu \sum_{|\alpha|=1} \int_{\mathbf{R}^{3}}|\nabla \xi||\Delta v|\left|D_{x}^{2 \alpha} \omega\right|\left|D_{x}^{\alpha} \omega\right|^{p-2} d x \\
\leq & \frac{\mu}{4 M} \sum_{|\alpha|=1} \int_{\mathbf{R}^{3}}\left|D_{x}^{2 \alpha} \omega\right|^{2}\left|D_{x}^{\alpha} \omega\right|^{p-2} d x \\
& +C \sum_{|\alpha|=1} \int_{\mathbf{R}^{3}}|\nabla \xi|^{2}|\Delta v|^{2}\left|D_{x}^{\alpha} \omega\right|^{p-2} d x \\
\leq & \frac{\mu}{4 M} \sum_{|\alpha|=0,1} \sum_{|\beta|=1} \int_{\mathbf{R}^{3}}\left|D_{x}^{\alpha} D_{x}^{\beta} \omega\right|^{2}\left|D_{x}^{\alpha} \omega\right|^{p-2} d x \\
& +C\|\nabla \xi(t)\|_{W_{p}^{1}\left(\mathbf{R}^{3}\right)}^{2}\|\Delta v(t)\|_{L_{p}\left(\mathbf{R}^{3}\right)}^{2}\|\omega(t)\|_{W_{p}^{1}\left(\mathbf{R}^{3}\right)}^{p-2} . \\
& \left|I_{6}\right| \leq\|\operatorname{rot} f(t)\|_{W_{p}^{1}\left(\mathbf{R}^{3}\right)}\|\omega(t)\|_{W_{p}^{1}\left(\mathbf{R}^{3}\right)}^{p-1} .
\end{aligned}
$$

Hence, using the inequality

$$
\sum_{|\alpha|=1}\left\|D_{x}^{\alpha} v(t)\right\|_{W_{p}^{1}\left(\mathbf{R}^{3}\right)} \leq C\|\omega(t)\|_{W_{p}^{1}\left(\mathbf{R}^{3}\right)}
$$

we have

$$
\begin{aligned}
& \frac{1}{p} \frac{d}{d t}\|\omega(t)\|_{W_{p}^{1}\left(\mathbf{R}^{3}\right)}^{p}+\frac{\mu}{2 M} \sum_{|\alpha|=0,1} \sum_{|\beta|=1} \int_{\mathbf{R}^{3}}\left|D_{x}^{\alpha} D_{x}^{\beta} \omega\right|^{2}\left|D_{x}^{\alpha} \omega\right|^{p-2} d x \\
& +\frac{\mu(p-2)}{M} \sum_{|\alpha|=0,1} \sum_{|\beta|=1} \int_{\mathbf{R}^{3}}\left|D_{x}^{\alpha} \omega \cdot D_{x}^{\alpha} D_{x}^{\beta} \omega\right|^{2}\left|D_{x}^{\alpha} \omega\right|^{p-4} d x \\
& \leq C\left[\|\omega(t)\|_{W_{p}^{1}\left(\mathbf{R}^{3}\right)}^{2}+\|\nabla \xi(t)\|_{W_{p}^{1}\left(\mathbf{R}^{3}\right)}\|\nabla p(t)\|_{W_{p}^{1}\left(\mathbf{R}^{3}\right)}+\|\nabla \xi(t)\|_{W_{p}^{1}\left(\mathbf{R}^{3}\right)}\|\omega(t)\|_{W_{p}^{1}\left(\mathbf{R}^{3}\right)}\right. \\
& \left.+\|\nabla \xi(t)\|_{W_{p}^{1}\left(\mathbf{R}^{3}\right)}^{2}\|\omega(t)\|_{W_{p}^{1}\left(\mathbf{R}^{3}\right)}+\|\operatorname{rot} f(t)\|_{W_{p}^{1}\left(\mathbf{R}^{3}\right)}\right]\|\omega(t)\|_{W_{p}^{1}\left(\mathbf{R}^{3}\right)}^{p-1}
\end{aligned}
$$

LEMMA 4.3. There exists $T_{0} \in(0, T]$ independent of $\mu$ such that 


$$
\sup _{0 \leq t \leq T_{0}}\left(\|\nabla \rho(t)\|_{W_{p}^{1}\left(\mathbf{R}^{3}\right)}+\|v(t)\|_{W_{p}^{2}\left(\mathbf{R}^{3}\right)}+\|\nabla p(t)\|_{W_{p}^{1}\left(\mathbf{R}^{3}\right)}\right) \leq C .
$$

Proof. Similarly to getting the estimate $\omega$, we first obtain

$$
\frac{1}{p} \frac{d}{d t}\|v(t)\|_{L_{p}\left(\mathbf{R}^{3}\right)} \leq C\left[\|\nabla p(t)\|_{L_{p}\left(\mathbf{R}^{3}\right)}+\|\nabla \xi(t)\|_{W_{p}^{1}\left(\mathbf{R}^{3}\right)}^{2}\|v(t)\|_{L_{p}\left(\mathbf{R}^{3}\right)}+\|f(t)\|_{L_{p}\left(\mathbf{R}^{3}\right)}\right] .
$$

Next, from the equation

$$
\operatorname{div}(\xi \nabla p)=\operatorname{div} f-\sum_{i, j=1}^{3} v_{x_{j}}^{i} v_{x_{i}}^{j}+\mu \nabla \xi \cdot \Delta v \equiv F
$$

we have

$$
\begin{aligned}
\|\nabla p(t)\|_{W_{p}^{1}\left(\mathbf{R}^{3}\right)} \leq & K_{5}\left(\|\nabla \xi(t)\|_{W_{p}^{1}\left(\mathbf{R}^{3}\right)}\right)\|F\|_{L_{p}\left(\mathbf{R}^{3}\right)} \\
\leq C & K_{5}\left(\|\nabla \xi(t)\|_{W_{p}^{1}\left(\mathbf{R}^{3}\right)}\right) \\
& \quad \times\left(\|\operatorname{div} f\|_{L_{p}\left(\mathbf{R}^{3}\right)}+\|\omega(t)\|_{W_{p}^{1}\left(\mathbf{R}^{3}\right)}^{2}+\|\nabla \xi(t)\|_{W_{p}^{1}\left(\mathbf{R}^{3}\right)}\|\omega(t)\|_{W_{p}^{1}\left(\mathbf{R}^{3}\right)}\right) .
\end{aligned}
$$

Therefore, if we set

$$
\begin{gathered}
Y(t)=1+\|\nabla \xi(t)\|_{W_{p}^{1}\left(\mathbf{R}^{3}\right)}+\|v(t)\|_{L_{p}\left(\mathbf{R}^{3}\right)}+\|\omega(t)\|_{W_{p}^{1}\left(\mathbf{R}^{3}\right)}, \\
B=\left(1+\|f\|_{C^{0}\left([0, T] ; W_{p}^{2}\left(\mathbf{R}^{3}\right)\right)}\right)^{2}
\end{gathered}
$$

then the above lemmas imply a differential inequality

$$
\frac{d}{d t} Y(t) \leq C B H(Y(t))
$$

where $H$ is a increasing function of $Y(t)$ independent of $\mu$.

Hence we conclude that $Y(t) \leq Z(t)$, where $Z(t)$ is the solution of the problem

$$
\left\{\begin{array}{l}
\frac{d}{d t} Z(t)=C B H(Z(t)), \\
Z(0)=Y(0),
\end{array}\right.
$$

and exists as a continuous function on an interval $\left[0, T_{0}\right]$ with $T_{0}>0$.

Since $T_{0}$ is obviously independent of $\mu$, we obtain the desired result.

4.2. Proof of Theorem 1.3. First, it follows from Theorems 1.1, 1.2 and Lemma 4.3 that the existence of a unique solution on $\left[0, T_{0}\right]$ with $T_{0}>0$ independent of $\mu$.

Next we prove (1.22). Subtracting (1.1) with $\mu>0$ from (1.1) with $\mu=0$, we get the following linear system of equations for $\sigma=\rho^{0}-\rho^{\mu}, w=v^{0}-v^{\mu}$ and $q=p^{0}-p^{\mu}$ : 


$$
\left\{\begin{array}{l}
\sigma_{t}+v^{\mu} \cdot \nabla \sigma=-w \cdot \nabla \rho^{0} \\
\rho^{\mu}\left[w_{t}+\left(v^{\mu} \cdot \nabla\right) w\right]+\nabla q=-\rho^{\mu}(w \cdot \nabla) v^{0}+\left(\nabla p^{0} / \rho^{0}\right) \sigma-\mu \Delta v^{\mu} \\
\operatorname{div} w=0 \\
\left.\sigma\right|_{t=0}=0 \\
\left.w\right|_{t=0}=0
\end{array}\right.
$$

In the same way for getting a priori estimates, we have, from (4.9),

$$
\begin{gathered}
\|\sigma(t)\|_{L_{p}\left(\mathbf{R}^{3}\right)} \leq C \int_{0}^{t}\|w(s)\|_{L_{p}\left(\mathbf{R}^{3}\right)} d s \\
\|w(t)\|_{L_{p}\left(\mathbf{R}^{3}\right)} \leq C \int_{0}^{t}\left(\|\sigma(s)\|_{L_{p}\left(\mathbf{R}^{3}\right)}+\|w(s)\|_{L_{p}\left(\mathbf{R}^{3}\right)}\right) d s+\mu C T_{0} .
\end{gathered}
$$

Hence, by Gronwall's inequality, we find that

$$
\|\sigma(t)\|_{L_{p}\left(\mathbf{R}^{3}\right)}+\|w(t)\|_{L_{p}\left(\mathbf{R}^{3}\right)} \leq \mu C T_{0} \exp \left(C T_{0}\right) .
$$

Furthermore, making use of the interpolation inequalities, we have

$$
\begin{aligned}
\|\nabla \sigma(t)\|_{L_{p}\left(\mathbf{R}^{3}\right)} & \leq C\|\sigma(t)\|_{L_{p}\left(\mathbf{R}^{3}\right)}^{1 / 2}\|\nabla \sigma(t)\|_{W_{p}^{1}\left(\mathbf{R}^{3}\right)}^{1 / 2} \\
& \leq C\|\sigma(t)\|_{L_{p}\left(\mathbf{R}^{3}\right)}^{1 / 2}\left(\left\|\nabla \rho^{0}(t)\right\|_{W_{p}^{1}\left(\mathbf{R}^{3}\right)}+\left\|\nabla \rho^{\mu}(t)\right\|_{W_{p}^{1}\left(\mathbf{R}^{3}\right)}\right)^{1 / 2} \\
& \leq C\|\sigma(t)\|_{L_{p}\left(\mathbf{R}^{3}\right)}^{1 / 2}, \\
\|\nabla w(t)\|_{L_{p}\left(\mathbf{R}^{3}\right)} & \leq C\|w(t)\|_{L_{p}\left(\mathbf{R}^{3}\right)}^{1 / 2}\|\nabla w(t)\|_{W_{p}^{1}\left(\mathbf{R}^{3}\right)}^{1 / 2} \\
& \leq C\|w(t)\|_{L_{p}\left(\mathbf{R}^{3}\right)}^{1 / 2}\left(\left\|\nabla v^{0}(t)\right\|_{W_{p}^{1}\left(\mathbf{R}^{3}\right)}+\left\|\nabla v^{\mu}(t)\right\|_{W_{p}^{1}\left(\mathbf{R}^{3}\right)}\right)^{1 / 2} \\
& \leq C\|w(t)\|_{L_{p}\left(\mathbf{R}^{3}\right)}^{1 / 2} .
\end{aligned}
$$

On the other hand, since $q(x, t)$ satisfies the equation

$$
\begin{aligned}
\operatorname{div}\left(\xi^{\mu} \nabla q\right)= & -\mu \nabla \xi^{\mu} \cdot \Delta v^{\mu}-\sum_{i, j=1}^{3}\left(v_{x_{j}}^{\mu, i} w_{x_{i}}^{j}+v_{x_{j}}^{0, i} w_{x_{i}}^{j}\right) \\
& +\Delta p^{0} \xi^{0} \xi^{\mu} \sigma+\nabla p^{0} \cdot \nabla \xi^{0} \xi^{\mu} \sigma+\nabla p^{0} \cdot \nabla \xi^{\mu} \xi^{0} \sigma+\nabla p^{0} \cdot \nabla \sigma \xi^{0} \xi^{\mu}
\end{aligned}
$$

we get

$$
\|\nabla q(t)\|_{W_{p}^{1}\left(\mathbf{R}^{3}\right)} \leq C\left(\|\sigma(t)\|_{W_{p}^{1}\left(\mathbf{R}^{3}\right)}+\|w(t)\|_{W_{p}^{1}\left(\mathbf{R}^{3}\right)}+\mu\right) .
$$

Thus, owing to (4.11), the proof of Theorem 1.3 is completed.

\section{References}

[1] S. Agmon, A. Douglis and L. Nirenberg, Estimates near the boundary for solution of elliptic partial differential equations satisfying general boundary conditions I, Comm. Pure Appl. Math. 17 (1959), 623-727. 
[2] H. Beirão da Veiga and A. Valli, On the Euler equations for nonhomogeneous fluids (I), Rend. Sem. Mat. Univ. Padova 63 (1980), 151-167.

[3] H. Beirão da Veiga and A. Valli, On the Euler equations for nonhomogeneous fluids (II), J. Math. Anal. Appl. 73 (1980), 338-350.

[4] H. Beirão da Veiga and A. Valli, Existence of $C^{\infty}$ solutions of the Euler equations for nonhomogeneous fluids, Comm. Partial Differential Equations 5 (1980), 95-107.

[5] S. Iтон, On the vanishing viscosity in the Cauchy problem for the equations of a nonhomogeneous incompressible fluid, Glasgow Math. J. 36 (1994), 123-129.

[6] O. A. LADYZHENSKAYA and V. A. Solonnikov, Unique solvability of an initial- and boundary-value problem for viscous incompressible nonhomogeneous fluids, J. Soviet Math. 9 (1978), 697-749.

[7] O. A. Ladyzhenskaya, V. A. SolonNikov and N. N. URAL'Ceva, Linear and Quasilinear Equations of Parabolic Type, Transl. Math. Monographs 23 (1968), Amer. Math. Soc.

[8] J. E. MARSDEN, Well-posedness of the equations of a non-homogeneous perfect fluid, Comm. Partial Differential Equations 1 (1976), 215-230.

[9] Н. Окамото, On the equations of nonstationary stratified fluid motion: Uniqueness and existence of the solutions, J. Fac. Sci. Univ. Tokyo 30 (1984), 615-643.

[10] V. A. SolonNikov, On boundary value problem for linear parabolic systems of differential equations of general form, Proc. Steklov Inst. Math. 83 (1965), 3-162.

[11] V. A. SolonNiKov, Estimates for solutions of nonstationary Navier-Stokes equations, J. Soviet Math. 8 (1977), 467-529.

[12] R. Temam, On the Euler equations of incompressible perfect fluids, J. Funct. Anal. 20 (1975), 32-43.

[13] A. VALLI and W. M. ZAJACZKowSKI, About the motion of nonhomogeneous ideal incompressible fluids, Nonlinear Anal. 12 (1988), 43-50.

Present Addresses:

SHIGeHARU ITOH

Department of Mathematics, Hirosaki University, Hirosaki, 036-8561 Japan.

Atusi TANI

Department of Mathematics, Keio University, Yokohama, 223-8522 Japan. 